Rev. Int. Contam. Ambie. 37, 167-185, 2021

https://doi.org/10.20937/RICA.53688

\title{
ENTROPÍA Y NEGUENTROPÍA: UNA APROXIMACIÓN AL PROCESO DE DIFUSIÓN DE CONTAMINANTES Y SU SOSTENIBILIDAD
}

\author{
Entropy and neguentropy: An approach to the process of pollutant diffusion and its sustainability \\ Patricio R. PACHECO HERNÁNDEZ ${ }^{1 *}$, Giovanni A. SALINI CALDERÓN ${ }^{2}$ \\ y Eduardo M. MERA GARRIDO ${ }^{1}$
}
${ }^{1}$ Departamento de Física, Facultad de Ciencias Naturales, Matemática y del Medio Ambiente, Universidad Tecnológica Metropolitana, Las Palmeras 3360, Ñuñoa, Santiago, Chile.
${ }^{2}$ Departamento de Matemática y Física Aplicadas, Facultad de Ingeniería, Universidad Católica de la Santísima Concepción, Alonso de Ribera 2850, Concepción, Chile.

*Autor para correspondencia: patricio.pacheco@utem.cl

(Recibido: agosto 2019; aceptado agosto 2020)

Palabras clave: contaminación ambiental, dinámica no lineal, calidad del aire, series temporales no lineales, caos.

\section{RESUMEN}

En este estudio se analizan series temporales de partículas gruesas y finas, de monóxido de carbono, humedad relativa, velocidad del viento y temperatura, de la ciudad de Santiago de Chile. Se realizaron medidas horarias de evolución de la concentración de contaminantes atmosféricos, las que fueron acompañadas con mediciones locales continuas, por sobre tres años, de temperatura, humedad relativa y velocidad del viento a nivel del suelo. Estas series surgen de mediciones efectuadas en seis estaciones de monitoreo durante el periodo de tiempo comprendido entre los años 2010 y 2013 (3.25 años). Su estudio, basado en análisis no lineal, permite obtener sus entropías de correlación a partir de las cuales se interpreta el proceso de difusión de los contaminantes a través del sistema Tierra-atmósfera. Como resultado, se muestra acuerdo pleno con las dinámicas de concentraciones observadas. Se discute como la neguentropía y el flujo asociado pueden propiciar la dinámica dispersiva, según las variables meteorológicas estudiadas, de los contaminantes emitidos acorde a su vida media particular. Se comprueba el carácter caótico de variables de contaminación como las partículas atmosféricas y algunas variables meteorológicas. Estas últimas podrían explicar por qué ciertas localidades en la ciudad de Santiago presentan menos contaminación por partículas que otras.

Key words: air pollution, nonlinear dynamics, air quality, nonlinear time series, chaos

\begin{abstract}
This paper presents a study of hourly air pollution time series of coarse and fine particulate matter, carbon monoxide, relative humidity, wind speed and temperature, from the city of Santiago de Chile. Hourly measurements were made of the evolution of the concentration of atmospheric pollutants, which were accompanied by continuous local measurements, for over three years, of temperature, relative humidity and wind speed
\end{abstract}


at ground level. These series arise from measurements made at six monitoring stations during the period between 2010 and 2013 (3.25 years). Its study, based on non-linear analysis, allows us to obtain its correlation entropies from which the process of diffusion of pollutants through the Earth-atmosphere system is interpreted. As a result, full agreement is shown with the dynamics of observed concentrations. It is discussed how neguentropy and associated flow can promote dispersive dynamics, according to the meteorological variables studied, of the pollutants emitted according to their particular half-life. It is discussed how neguentropy and associated flow can promote dispersive dynamics, according to the meteorological variables studied, of the pollutants emitted according to their particular half-life. The chaotic character of pollution variables is checked as particulate matter and some meteorological variables. These last variables could explain why certain localities in the city of Santiago have less pollution by particulate matter than others.

\section{INTRODUCCIÓN}

La exposición a los contaminantes del aire trae consigo problemas respiratorios, cardiovasculares y recientemente se está pensando que partículas minúsculas se pueden alojar en el cerebro humano, siendo un factor para el desarrollo de enfermedades neurodegenerativas (Araujo 2011). De acuerdo con la OMS, una de cada ocho personas en el mundo muere por contaminación ambiental (OMS 2018). La contaminación atmosférica consiste en la liberación en grandes cantidades de elementos nocivos que producen daño al ser humano y a su hábitat. Esta contaminación puede alcanzar concentraciones superiores al nivel considerado normal. Por su parte, los aerosoles atmosféricos, o partículas atmosféricas, pueden ser sólidas o líquidas (Toledano 2008) y se clasifican, de acuerdo con su tamaño, en aquellas de diámetro igual o menor a $10 \mu \mathrm{m}\left(\mathrm{PM}_{10}\right)$, y las de diámetro igual o menor a $2.5 \mu \mathrm{m}\left(\mathrm{PM}_{2.5}\right)$. Como las $\mathrm{PM}_{2.5}$ están contenidas en las $\mathrm{PM}_{10}$, podemos decir que las primeras son la fracción fina de las $\mathrm{PM}_{10}$. Las partículas con diámetro entre $2.5 \mu \mathrm{m}$ y $10 \mu \mathrm{m}$ serían la fracción gruesa de las $\mathrm{PM}_{10}$. Pero también existe una fracción ultrafina e incluso nanométrica. Actualmente, hay estudios que muestran el daño que la contaminación por estas partículas provoca en la salud humana (Ilabaca et al. 1999, Belcik et al. 2018, Jia et al. 2017) debido a que poseen propiedades mutagénicas o carcinogénicas, pudiendo ocasionar alteraciones a nivel de la población (Schwartz et al. 2002, Raabe 2005, Reyna et al. 2018). Las partículas cuyo diámetro sea mayor a $5 \mu \mathrm{m}$ se depositan en las vías aéreas superiores, en la tráquea y los bronquios, y aquellas cuyo diámetro es menor a dicho valor tienen mayor probabilidad de depositarse en los bronquiolos y alveolos (Peters y Dockery 2005), e inclusive la fracción fina puede llegar hasta la sangre (Cifuentes et al. 2000), provocándose incluso problemas cardiovasculares (Lee et al 2014).

El desarrollo urbano, el incremento del parque automotor y la creciente industrialización llevan consigo al aumento de la contaminación ambiental, fenómeno observado en todo el mundo. La contaminación atmosférica en la ciudad de Santiago se ha estudiado desde hace bastante tiempo (Morales 2006), siendo considerado como un problema endémico, al igual que en grandes urbes del mundo (Molina y Molina 2004). Esta ciudad ya fue declarada como zona saturada (Biblioteca del Congreso Nacional decreto 131, 1996), estableciéndose que está sobrepasada en cuatro contaminantes atmosféricos: partículas atmosféricas $\left(\mathrm{PM}_{10} / \mathrm{PM}_{2.5}\right)$, ozono $\left(\mathrm{O}_{3}\right)$, monóxido de carbono $(\mathrm{CO})$ y partículas en suspensión (PST). Debido a los planes de prevención y descontaminación ambiental (PPDA) aplicados a la Región Metropolitana, y posteriormente en el año 2000 el plan de transporte urbano (PTUS), se ha logrado disminuir los niveles de contaminación de manera notable (MMA 2019). Por ejemplo, desde 37 preemergencias (días en los que las PM exceden $\operatorname{los} 240 \mu \mathrm{g} / \mathrm{m}^{3}$ ) en 1997, a sólo dos en el año 2004. No obstante, debido al modo de vida moderno, en los últimos años la contaminación ha vuelto a aumentar. Así, en el año 2008 hubo 14 alertas y 6 preemergencias. Pero también está la mala calidad del aire de ciudades regionales (o ciudades de tamaño medio) al sur de Chile como, Coyhaique, Osorno, Temuco, Chillán, Talca, Rancagua, entre otras, debido fundamentalmente al uso de leña húmeda empleada como calefacción de viviendas (Salini 2014).

\section{Conceptos fundamentales}

De acuerdo con Prigogine, desde el momento en que en un sistema se produce un pequeño alejamiento del equilibrio termodinámico, coexisten el orden y 
desorden (Prigogine 1967). Además, según este autor, el no equilibrio es creador de estructuras denominadas disipativas puesto que sólo existen lejos del equilibrio, requiriendo de cierta disipación de energía para su sobrevivencia y, por tanto, el mantenimiento de una interacción con el exterior. De forma análoga a la de una ciudad, que sólo existe en cuanto a su funcionamiento y manutención de intercambios con el exterior, una estructura disipativa desaparece cuando deja de ser "alimentada". Es justamente este concepto introducido por Prigogine, el que nos sirve de base para suponer una de nuestras hipótesis de trabajo: que los contaminantes se comportarían de manera análoga a una estructura disipativa, siendo alimentada por el mismo ser humano, al estar emitiendo partículas y otros contaminantes a la atmósfera. En otras palabras, es el mismo ser humano el que está "sustentando" a la contaminación del aire. Situación que no se revertiría si no fuese por la interacción generada por los parámetros meteorológicos presentes en el sistema Tierra-atmósfera.

Para que se forme una estructura disipativa, en principio, se tienen unas condiciones de equilibrio (sean cuales sean éstas) en el que se producen fluctuaciones respecto de dichas condiciones que con el paso del tiempo se amortiguan (Fig. 1). Si se va aportando energía, las fluctuaciones van siendo más grandes pero el sistema aún consigue amortiguarlas. Hasta que llega un momento en el que se sobrepasa un umbral. La fluctuación ya no se amortigua, sino que se estabiliza en un estado alejado del equilibrio primitivo, pero igualmente estable mientras continúe

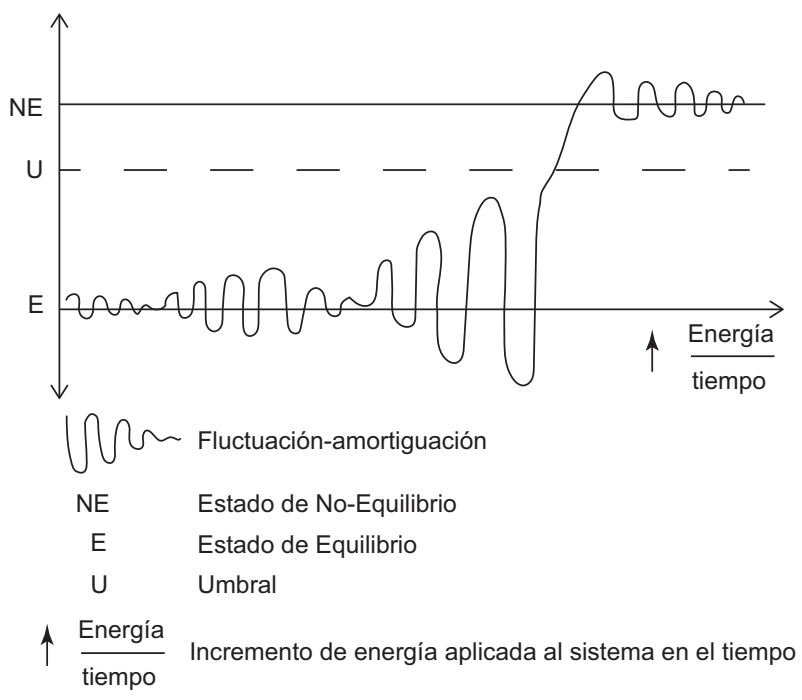

Fig. 1. Incremento de la energía aplicada a un sistema, en función del tiempo. Formación de una estructura disipativa. el aporte de energía: se ha formado una estructura disipativa (Manríquez 1987).

La ecuación de balance de entropía (Ruelle 2003, Cuesta 2006) se deriva de la relación de Gibbs (Liu et al. 2011), de la suposición de equilibrio local, tal que la entropía por unidad de volumen, s, se escribe en la forma:

$\frac{\partial s}{\partial t}=-\nabla \cdot \vec{J}_{s}+\sigma$

donde $\nabla \cdot \vec{J}_{s}$ es la divergencia del flujo de entropía por unidad de volumen a través de la superficie cuyo contorno encierra al sistema (para mayor referencia ver Anexo I). En la ecuación (1), denota la producción de entropía y $\vec{J}_{s}$ es el flujo de entropía definido como (Liu et al. 2011):

$\vec{J}_{s}+s \vec{v}+\frac{1}{T} \vec{J}_{q}-\sum_{k} \frac{\mu_{k}}{T} \vec{J}_{k}$

donde $s=S / V$, es la entropía por unidad de volumen para una densidad total $\rho, \vec{v}$ es la representación vectorial de la velocidad, $\vec{J}_{q}$ el flujo de calor (Onsager 1931), T es la temperatura en la escala absoluta, es el potencial químico para la k-ésima especie componente (medido en Gibb/mol) y $\vec{J}_{k}$ el flujo difusivo de masa de la k-ésima especie componente.

El sistema Tierra-atmósfera intercambia radiación con el espacio, lo cual conduce y mantiene casi todos los procesos dentro de este sistema. Según Liu (Liu et al. 2011), la esencia del intercambio de radiación de la Tierra con el espacio es que los fotones de alta energía con baja entropía provenientes del Sol, ingresan al sistema Tierra-Atmósfera (Stephens y O’Brein 1993, Liu et al. 2011), y los fotones de baja energía con alta entropía son emitidos desde el sistema Tierra-Atmósfera al espacio, lo que se muestra en la figura 2. Obsérvese, además, que en ninguna parte de esta figura se señala la cantidad de radiación ni tampoco cuál es más importante, más bien, indica en qué región la radiación es más intensa. En general, el sistema Tierra-atmósfera puede pensarse como un sistema multicuerpo que es cerrado al intercambio de material que no sea de radiación y abierto a intercambio de radiación con el espacio. Para semejante sistema complejo, el flujo neto de entropía que resulta del intercambio de radiación restringe la proporción de producción de entropía interna del sistema Tierra-atmósfera, una medida fundamental de todas las actividades dentro de este sistema, que incluye procesos oceánicos, atmosféricos y biológicos (Katchalsky y Curran 1965, Glansdorff y Prigogine 1971, Mahulikar y Herwig 2009, Torday 2016, Haddad 2017). 


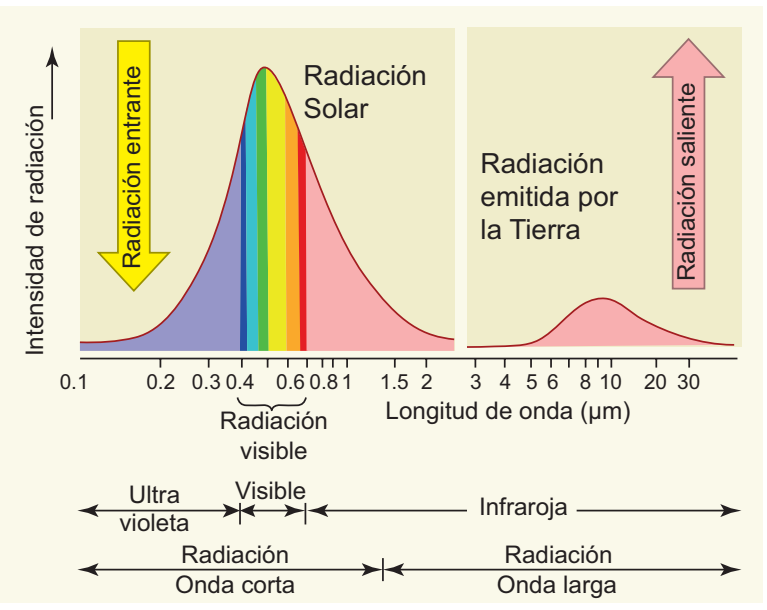

Fig. 2. Espectro de radiación electromagnética entrante y saliente al sistema tierra-atmósfera. Nótese que la abscisa es la longitud de onda de la radiación y la ordenada es su intensidad.

Como la entropía en un sistema es determinante en el orden del sistema según su extensión, la evolución de un sistema atmosférico reducido debería relacionarse con el flujo de entropía (Liu et al. 2011). La idea de sistema reducido apunta a que, en el modelo de capas aplicado, se supone a los contaminantes como una capa externa (de entropía $\Delta \mathrm{S}_{\mathrm{E}}$ ) que abraza a la capa interna de la atmósfera próxima al suelo (de entropía $\Delta \mathrm{S}_{\mathrm{I}}$ ). Esto es razonable dado que, en general, los contaminantes son emitidos a altas temperaturas y por convección empujan hacia abajo el aire atmosférico más frío, aprisionándolo, estimulando aún más las condiciones para una interacción de contaminantes y variables meteorológicas que propician el flujo de entropía.

En 1900, investigaciones de Bénard (Bénard 1900, 1901) en fluidos delgados y estratificados, originalmente en reposo, con una superficie libre a baja temperatura, $T_{1}$, la que es calentada desde la base con una temperatura alta, $\mathrm{T}_{2}$, le hicieron notar un patrón regular de celdas convectivas hexagonales, las que se organizaban abruptamente cuando la diferencia de temperatura $\left(\mathrm{T}_{2}-\mathrm{T}_{1}\right)$ alcanzaba el umbral de inestabilidad primaria (De Groot y Mazur 1962, Glansdorff y Prigogine 1971, Eckert et al. 1998), sugiriendo que cada sistema que obtiene calor de un entorno a una temperatura más alta perderá calor en un entorno a una temperatura más baja, experimentando un cambio neto de entropía negativa o neguentropía, tal como se ilustra en la figura 3 (Liu et al. 2011). En este caso, el cambio de entropía $\left(\delta \mathrm{S}_{\mathrm{e}}\right)$ de un estado de equilibrio a otro estado - también de equilibrio-, cuyo proceso es irreversible está dado por (Dewar 2005):

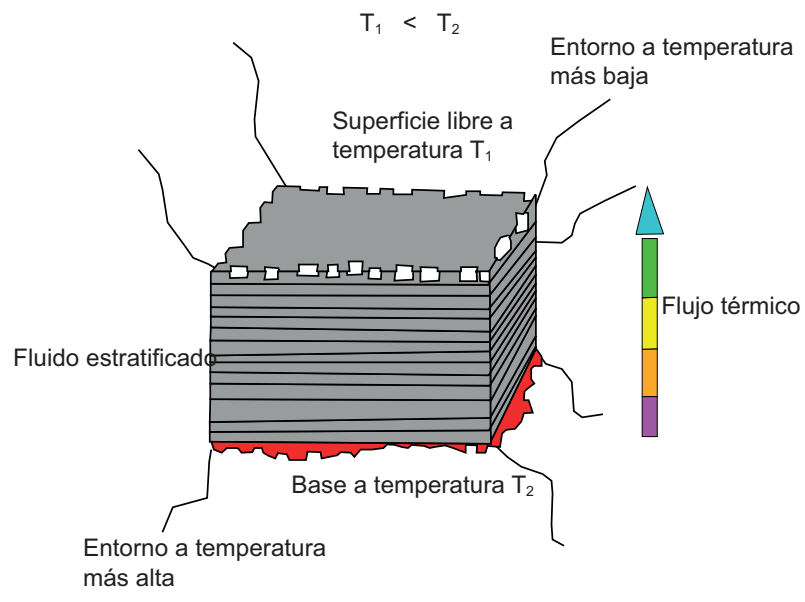

Fig. 3. Representación esquemática de un fluido estratificado con flujo térmico.

$\delta S_{e}=\frac{Q_{2}}{T_{2}}-\frac{Q_{1}}{T_{1}}=\frac{T_{1}-T_{2}}{T_{1} T_{2}} Q<0$

donde $Q_{1}$ y $Q_{2}$ son flujos térmicos a través de la parte superior y la base del fluido estratificado, respectivamente. $Q$ es el flujo cuando el sistema alcanza el estado estable $Q=Q_{1}=Q_{2}$. Schrödinger señala -en 1940- que el flujo de neguentropía es muy significativo para un sistema (viviente o no viviente). Esto es, la existencia de la vida dependerá de una ganancia continua de neguentropía de sus alrededores (Schrödinger 1944). Es decir, un flujo negativo de entropía hará que un sistema inicialmente en equilibrio -O incluso en reposo- se organice. O, por otro lado, conducirá a un sistema, en estado de no equilibrio a un estado más alejado del equilibrio (haciéndolo que se fortalezca). (Sinai 1959, De Groot y Mazur 1962, Liu et al. 2011).

Para un conjunto de mediciones localizadas, de variables meteorológicas y contaminantes, constituidas en series de tiempo comprobada su característica caótica, el cálculo de los flujos entrópicos, $\partial \mathrm{s} / \partial \mathrm{t}$ permite analizar la información termodinámica. Esto no es contradictorio con la segunda ley de la termodinámica de modo tal que el cambio de entropía total (sistema más alrededores) sea siempre positivo o igual a cero. Para este estudio, de primera aproximación, se subentiende que esto es sólo cierto cuando las fronteras de los alrededores sean adiabáticas y rígidas, es decir, el sistema interno más el sistema externo constituyen un sistema cerrado En este caso $\Delta$ $S \geq 0$, en donde la desigualdad corresponde a un proceso natural o irreversible y la igualdad a un proceso reversible. Así el sistema interno puede experimentar 
un decremento de entropía (por ser abierto) a costa del incremento de entropía del sistema externo (por ser este último un sistema cerrado que contiene en su seno al sistema en cuestión).

\section{La entropía de Kolmogorov y su relación con la pérdida de información}

De acuerdo con Farmer, se tiene que una de las diferencias esenciales entre el comportamiento caótico y el predecible es que las trayectorias caóticas generan continuamente nueva información mientras que las trayectorias predecibles no lo hacen (Farmer 1982, Farmer et al. 1983). La entropía métrica hace esta noción más rigurosa. Además de proporcionar una buena definición de caos, la entropía métrica proporciona una forma cuantitativa para describir cuán caótico es un sistema dinámico.

En la entropía de Kolmogorov (Martínez y Vinagre 2016), $S_{K}$, es el promedio de pérdida de información (Shannon 1948, Brillouin 1962) cuando "l" (lado de la celda en unidades de información) y $\tau$ (tiempo) se hacen infinitesimales:

$$
S_{K}=-\lim _{\tau \rightarrow 0} \lim _{l \rightarrow 0} \lim _{n \rightarrow \infty} \frac{l}{n \tau} \sum_{0 \ldots . n} P_{o \ldots . n} \log P_{0 \ldots . n}
$$

Se expresa en bits de información por segundo y bits por iteración para un sistema discreto (Shaw 1981, Cohen y Procaccia 1985). El orden de prioridad en el cálculo de los límites es tal cual aparece en ecuación (4). Primero $n \rightarrow \infty$, en seguida $l \rightarrow 0$, para eliminar la dependencia de la partición elegida. En la ecuación (4), $n$ es el número de celdas o particiones. Finalmente, $\tau \rightarrow 0$, que sólo es necesario en sistemas continuos.

La diferencia de entropía de Kolmogorov, $\Delta \mathrm{S}_{\mathrm{K}}$ $=\mathrm{S}_{\mathrm{Kn}+1}-\mathrm{S}_{\mathrm{Kn}}$ entre una celda y otra representa la información adicional que se necesita para saber en qué celda $\left(i_{n}+1\right)$ se va a encontrar el sistema en el futuro. Por lo tanto, esta diferencia mide la pérdida de información del sistema en el transcurso del tiempo.

En resumen, para el cálculo de la entropía de Kolmogorov, primero se comprueba que la entropía se encuentre entre cero e infinito $\left(0<\mathrm{S}_{\mathrm{K}}<\right)$, lo cual permite verificar la presencia de un comportamiento caótico. Si la entropía de Kolmogorov es igual a 0, no se pierde información y el sistema es regular y predecible. $\mathrm{Si} \mathrm{S}_{\mathrm{K}}=\infty$, el sistema es totalmente aleatorio y es imposible hacer cualquier predicción. En segundo lugar, se determina la cantidad de información que se requiere para predecir el comportamiento futuro de dos sistemas interactivos, en este caso, la atmósfera y los contaminantes. Luego se calcula la velocidad con la que el sistema pierde (o desactualiza) información en el tiempo. Finalmente, se establece el horizonte máximo de predictibilidad del sistema, aquella frontera a partir de la cual no se puede realizar predicción alguna, ni tampoco elaboración de nuevos escenarios (Kolmogorov 1959).

\section{Condiciones de flujo entrópico}

Para todo sistema termodinámico abierto la entropía total, $\Delta \mathrm{S}$, se puede escribir como la suma de la entropía interna del sistema, $\Delta \mathrm{S}_{\text {INTERNA }}\left(\Delta \mathrm{S}_{\mathrm{I}}\right)$, y la entropía externa a él, $\Delta \mathrm{S}_{\text {EXTERnA }}\left(\Delta \mathrm{S}_{\mathrm{E}}\right)$ (LópezCorona et al. 2016):

$\Delta S=\Delta S_{\text {interna }}+\Delta S_{\text {Externa }} \geq 0$

Desde el punto de vista de este estudio, $\Delta \mathrm{S}_{\mathrm{I}}$ es la entropía interna o natural del sistema Tierra-atmósfera y $\Delta \mathrm{S}_{\mathrm{E}}$ es la entropía asociada a los contaminantes, lo que es explicado en el apartado de Resultados. La producción de entropía - expresada como variación en el tiempo- está dada como:

$\frac{d S}{d t}=\frac{d S_{l}}{d t}+\frac{d S_{E}}{d t}$

La segunda ley de la termodinámica establece que la producción interna de entropía debido a procesos irreversibles que ocurren en su interior -aplicado al sistema Tierra-atmósfera- es:

$\frac{d S_{I}}{d t}>0$

Para el caso especial donde las perturbaciones son constantes, la teoría termodinámica clásica irreversible establece que eventualmente el sistema alcanzará un estado termodinámico estacionario en el cual todas las variables microscópicas, incluyendo la entropía total, son estacionarias en el tiempo. Vale decir, si

$\frac{d S}{d t}=0$

entonces,

$\frac{d S_{I}}{d t}=-\frac{d S_{E}}{d t} \Rightarrow \frac{d S_{E}}{d t}<0$

que tiene las características de un flujo de entropía negativa (neguentropía).

\section{MATERIALES Y MÉTODOS}

\section{Área de estudio}

La ciudad de Santiago de Chile, se sitúa a $33.5^{\circ}$ Sur y $70.8^{\circ}$ Oeste. Posee una población de 7508334 
habitantes que representa el $40 \%$ de la población del país, en una superficie de alrededor de $135 \mathrm{~km}^{2}$. Está ubicada en el centro del país, a una altura aproximada de $520 \mathrm{~m} \mathrm{snm}$. La altitud por sobre el nivel del mar crece de poniente a oriente. Está rodeada por dos sistemas montañosos: la cordillera de los Andes y la cordillera de la Costa.

Su clima es de tipo mediterráneo, siendo los meses diciembre a febrero calurosos y secos, alcanzando temperaturas máximas alrededor de $35^{\circ} \mathrm{C}$, a la sombra. Debido a su topografía y a las condiciones meteorológicas imperantes, especialmente en otoño e invierno, se observa por lo general una mala dispersión, tanto horizontal como vertical, de contaminantes emitidos por numerosas fuentes en la ciudad. Las emisiones tienen también una tendencia al aumento por la creciente densidad poblacional, lo que lleva asociado un aumento de fuentes fijas y móviles. Además, el parque automotor ha estado creciendo significativamente durante los últimos años.

Existen dos fenómenos meteorológicos que obstaculizan la adecuada dispersión de los contaminantes en esta ciudad. Estos son la inversión térmica de subsidencia y la radiativa. La inversión térmica se define como aquel fenómeno en el que se asienta una capa de aire cálido por sobre masas de aire más frías, impidiendo así el flujo del aire en dirección vertical (corriente ascendente), estabilizando las condiciones e impidiendo, por tanto, la dispersión vertical de los contaminantes. La inversión térmica de subsidencia es aquel fenómeno que se presenta por el predominio de altas presiones en el Pacífico (anticiclón), lo cual va acompañado por el descenso de aire sobre la región metropolitana, que al comprimirse se calienta rompiendo la tendencia del gradiente típico de temperatura. Se forma así una capa de inversión cuya base se puede situar a alturas menores a los $700 \mathrm{~m}$ snm. En cambio, la inversión térmica radiativa se produce por el enfriamiento de la tierra en períodos fríos -especialmente noches de invierno-, afectando a las masas de aire inmediatamente contiguas a ella, que quedan debajo de las capas más cálidas. Se ha logrado establecer que en la región de Santiago se dan dos tipos de condiciones sinópticas ligadas directamente con los episodios de alta contaminación atmosférica, a las cuales se les denomina tipo A y tipo BPF (Rutland y Garreaud 1995).

\section{Los datos}

Las series de tiempo de concentraciones horarias de contaminantes y variables meteorológicas fueron recopiladas desde la Red MACAM III, mantenida por el Sistema de Información Nacional de Calidad del Aire (SINCA) dependiente del Ministerio del Medioambiente de Chile (MMA), desde el año 2010 hasta el año 2013. Esto corresponde a 3.25 años o 39 meses. Las variables de contaminación fueron $\mathrm{PM}_{10}$, $\mathrm{PM}_{2.5}$ y CO. A su vez, las variables meteorológicas consideradas fueron temperatura $(\mathrm{T})$, humedad relativa (HR) y magnitud de la velocidad del viento (VV). En total, son 28464 datos por cada variable. En el cuadro I se muestra la estadística descriptiva para $\mathrm{PM}_{2.5}$. Las estaciones de monitoreo que se usaron en este estudio fueron seis: La Florida (EML), Las Condes (EMM), Santiago-Parque O'Higgins (EMN), Pudahuel (EMO), Puente Alto, (EMS) y Quilicura (EMV) (Fig. 4). En figura 5 se puede observar la serie temporal para la estación Pudahuel (EMO), durante el período en estudio. Las otras estaciones presentan gráficas similares.

Es común hallar en este tipo de series datos faltantes, que puede deberse a diferentes factores (Junninen et al. 2004, Norazian et al. 2008, Junninen et al. 2004, Salini y Medina 2017). Éstos se rellenaron mediante la técnica de kriging (Emery 2005, Asa et al. 2012). En particular, esta técnica, que se ha aplicado para

CUADRO I. ESTADÍSTICA DESCRIPTIVA DE PARTÍCULAS ATMOSFÉRICAS PM 2.5 (HORARIA Y ANUAL) EN PARA CADA ESTACIÓN DE MONITOREO (2010 a 2013)

\begin{tabular}{ccccccr}
\hline $\begin{array}{c}\text { Estación } \\
\text { monitoreo }\end{array}$ & Media & $\begin{array}{c}\text { Valor* } \\
\text { máximo }\end{array}$ & $\begin{array}{c}\text { Valor } \\
\text { mínimo }\end{array}$ & $\begin{array}{c}\text { Desviación } \\
\text { estándar }\end{array}$ & $\begin{array}{c}\text { Coeficiente } \\
\text { asimetría }\end{array}$ & Curtosis \\
\hline EML & 25.67 & 168 & 1 & 17.41 & 1.83 & 4.79 \\
EMM & 20.14 & 133 & 1 & 11.81 & 1.30 & 3.09 \\
EMN & 25.79 & 230 & 1 & 18.61 & 1.83 & 4.95 \\
EMO & 26.33 & 365 & 1 & 26.33 & 3.82 & 22.94 \\
EMS & 25.95 & 235 & 1 & 25.95 & 1.79 & 5.84 \\
EMV & 19.26 & 358 & 1 & 25.25 & 2.58 & 13.47 \\
\hline
\end{tabular}

* Todos los valores son en $\mu \mathrm{g} / \mathrm{m}^{3}$ 


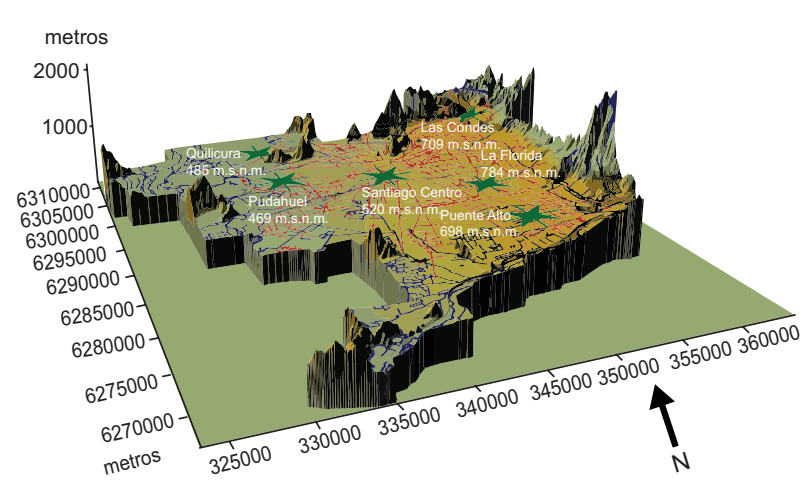

Fig. 4. Localización de las estaciones de monitoreo en estudio para la ciudad de Santiago de Chile.

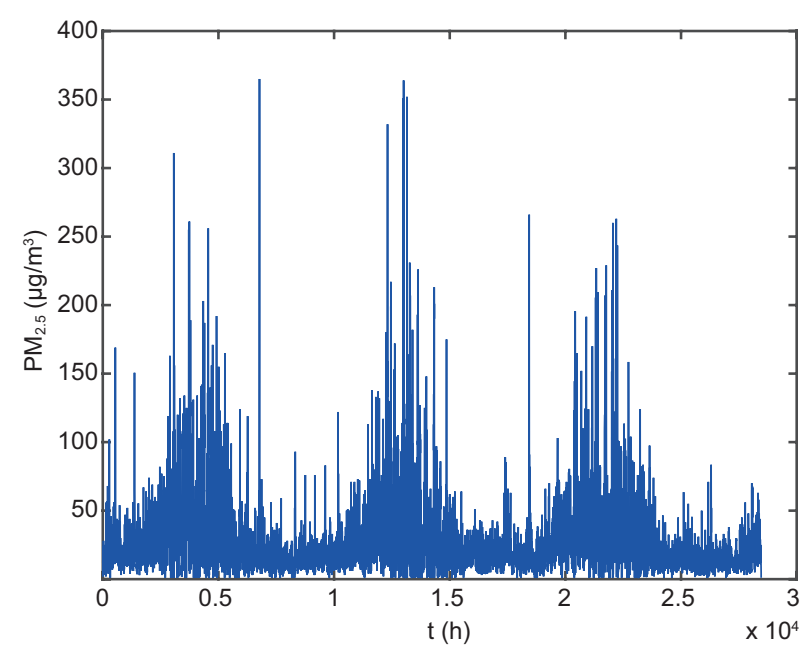

Fig. 5. Serie temporal de $\mathrm{PM}_{2.5}$ (estación EMO-Pudahuel).

este caso, es el método geoestadístico espacio temporal que proporciona un marco de trabajo probabilístico para el análisis de datos y predicciones, que se basa en la dependencia conjunta espacial y temporal entre las observaciones (Kyriakidis y Journel 1999). El análisis se centra en la interpolación espacial en instantes de tiempo específicos, comparándose los diferentes mapas en el tiempo, además, de en el modelado de series de tiempo múltiples donde cada ubicación espacial está asociada con una serie de tiempo diferente (Ramos et al. 2016, Mera et al. 2018). La teoría centrada en la predicción geoestadística muestra, además, la dimensión del tiempo (Cressie y Huang 1999).

Así, esta técnica permite interpolar la variable a medir faltante (concentración de contaminante) en una estación de datos (coordenadas espaciales definidas), en función de la información similar presente en estaciones de monitoreo espacialmente próximas (vecinas), para un intervalo de tiempo análogo. Cabe destacar que claramente no se transgrede ningún principio estadístico básico.

Este estudio pretende demostrar que los contaminantes de origen antrópico se comportan de manera similar a una estructura disipativa, siendo alimentada por el mismo ser humano, al estar emitiendo partículas y otros contaminantes a la atmósfera, originados en la ciudad de Santiago de Chile. Además, pretende verificar su origen no lineal, así como su carácter caótico. A su vez, muestra la situación actual de este contaminante, especialmente en época invernal y busca ser una contribución para llevar a cabo estudios más detallados, aplicables a otras localidades.

\section{Herramientas de análisis para series temporales no lineales}

Para el análisis y predicción de series temporales los métodos lineales pueden ser el punto de partida en el estudio del comportamiento de datos de contaminación del aire y meteorológicos. No obstante, se consiguen resultados más relevantes mediante el uso de herramientas no lineales (Kantz y Schreiber 2004, Sivakumar et al. 2007). Esto se debe a que, en este tipo de datos, pequeñas causas generan grandes efectos en los valores a futuro (Lorenz 1963), lo que es la esencia de la no linealidad (dependencia sensible de condiciones iniciales o efecto "mariposa") y aún más, caos en su evolución (Kumar et al. 2008, Lee y Lin 2008, Salini y Pérez 2015).

El estudio de una serie temporal no lineal comienza con la determinación de dos parámetros importantes: el retardo temporal, $\tau$ y la dimensión de encajamiento, $m$ (también llamada, inserción o "embedding", $d_{e}$ ) y se realiza empleando el método debido a Takens (1981). Para ello, se usa la información mutua promedio (Fraser y Swinney 1986) -en el primer caso- y el método de falsos vecinos más próximos -en el segundo- (Salini y Pérez 2006, Salini y Pérez 2015). Otros índices de relevancia en el análisis no lineal son el exponente de Lyapunov, 1 (Eckmann et al. 1986), la dimensión de correlación, DC (Sprott 2003), el exponente de Hurst, H (Grassberger y Procaccia 1983), la entropía de Kolmogorov, $\mathrm{S}_{\mathrm{K}}$ y la entropía de correlación, $\mathrm{K}_{2}$.

El exponente de Lyapunov se define como $\lambda$ y a partir de la ecuación (Salini y Pérez 2015):

$\lambda=\frac{1}{N} \operatorname{In} \frac{d_{n}}{d_{0}}$

siendo $\mathrm{N}$ el número de iteraciones. Vale decir, si dos puntos de una órbita están muy próximos inicialmente, 
se calcula este exponente para $\mathrm{N}$ grande. Si luego de $\mathrm{N}$ iteraciones los puntos se separan, habrá indicio de posible caos en dicho sistema. Un valor positivo del máximo exponente de Lyapunov es indicio de caos (Kantz y Schreiber 2005). Para una serie de tiempo dada, la suma de todos los exponentes positivos de Lyapunov define su entropía $\mathrm{S}_{\mathrm{K}}$ y su recíproco el tiempo promedio de predictibilidad, $\mathrm{T}_{\mathrm{P}}=1 / \mathrm{S}_{\mathrm{K}}($ Salini y Pérez 2015).

En la práctica el exponente de Lyapunov se obtiene a partir de esta ecuación en el límite de $\mathrm{N}$ grande, para el cual la saturación comienza a ser evidente. Por lo mismo, y por condiciones de estabilidad en su cálculo, se debe tener -idealmente- sobre 5000 datos (Wolf et al.1985).

Por su parte, la entropía de correlación, $\mathrm{K}_{2}$ (Gao et al 2007; Sprott 2003) se define como:

$\mathrm{K}_{2}=\lim _{m \rightarrow \infty} \lim _{\tau \rightarrow 0} \lim _{n \rightarrow \infty} \log \frac{C(m, r)}{C(m+1, r)}$

donde $\mathrm{n}$ es el número de puntos $-\mathrm{o}$ datos-, $\mathrm{m}$ es la dimensión de encajamiento y $\mathrm{r}$ es el radio del círculo o hiperesfera. $\mathrm{K}_{2}$ es cero, positivo o infinito para datos regulares, caóticos o aleatorios, respectivamente. Entonces, esta entropía -al igual que la de Kolmogorov- permite determinar si una serie temporal de datos experimentales es regular, caótica o aleatoria, tanto para los contaminantes como para las variables meteorológicas.

La ecuación (10), C(m, r) es la suma de correlación de la trayectoria reconstruida de una serie temporal. Un método para estimar $\mathrm{K}_{2}$ en datos experimentales está basado en Grassberger y Procacia (1983). En nuestro caso, se ha calculado sólo la entropía de Kolmogorov, $\mathrm{S}_{\mathrm{K}}$ a las series de contaminantes y variables meteorológicas, y pueden verse los resultados en cuadro II.

$\mathrm{C}(\mathrm{m}, \mathrm{r})$ es la suma de correlación para una dimensión de encajamiento $\mathrm{m}$ dada y se usa para estimar la dimensión de correlación (Sprott 2003). Se define a partir de:

$$
\begin{gathered}
C(r)=\frac{2}{n(\mathrm{n}-1)} \sum_{j=1}^{n} \sum_{i=j+i}^{n} H\left(r-r_{i j}\right)= \\
\lim _{n \rightarrow \infty} \frac{2}{n(\mathrm{n}-1)} \sum_{i \neq \mathrm{j}}^{n} H\left(r-\left\|X_{i}-X_{j}\right\|\right)
\end{gathered}
$$

$\mathrm{C}(\mathrm{r})$ es la integral (o función) de correlación y se puede interpretar como el número de puntos dentro de todos los círculo de radio r normalizado a 1 , cuando $r$ es suficientemente grande que incluya todos los puntos sin doble conteo; $n$ es el número de datos; $\mathrm{H}$ es la función de Heaviside (o función escalón), \|..\| denota la norma o distancia entre dos vectores, siendo la distancia euclidiana la más usada porque entrega resultados más robustos en presencia de ruido, y r un número real que debe escogerse con mucho cuidado, ya que valores pequeños de $r$ hacen que $C(r)$ no tenga sentido, y para valores grandes de $\mathrm{r}, \mathrm{C}(\mathrm{r})$ no entrega información útil.

La ecuación (11) también puede escribirse como:

$$
\begin{aligned}
& C(r)=\lim _{n \rightarrow 0} \frac{1}{n^{2}} \\
& \text { [número de pares }\left(x_{i}, x_{j}\right) \text { tales que }\left|x_{i}, x_{j}\right|<\mathrm{r} \text { ] }
\end{aligned}
$$

Se calcula $C(r)$ haciendo variar $r$ desde 0 hasta el valor más grande posible de $x_{i}-x_{j}$. Para valores suficientemente pequeños de $\mathrm{r}$ y para una cantidad de datos muy grande, $\mathrm{C}(\mathrm{r})$ se comporta siguiendo una ley de potencias del tipo:

$$
C(r) r^{D_{c}}
$$

$\mathrm{D}_{\mathrm{C}}$ es la dimensión de correlación (o exponente de correlación). A partir de ecuación (13) y tomando logaritmo en ambos lados, vemos que $\mathrm{D}_{\mathrm{C}}$ se obtiene al hacer un gráfico $\log C(r)$ vs. $\log$ r. Se observa que, si $D_{C}$ no se satura en algún valor a medida que $\mathrm{m}$ crece, el proceso es aleatorio (o estocástico). En caso contrario, si $\mathrm{D}_{\mathrm{C}} \mathrm{se}$ satura en algún valor, se dice que la serie temporal es más bien determinista (Chelani y Devotta 2006). En nuestro estudio, para las seis series temporales, $\mathrm{D}_{\mathrm{C}} \mathrm{se}$ saturó en los valores dados en el cuadro III. Una dimensión de correlación, $\mathrm{D}_{\mathrm{C}}>5$ implica esencialmente datos aleatorios, lo que no ocurre en nuestro estudio.

Uno de los objetivos de esta investigación es examinar el comportamiento (determinista, caótico o aleatorio) de tres series de tiempo de contaminantes $\left(\mathrm{PM}_{10}, \mathrm{PM}_{2.5}\right.$ y CO$)$ y de tres series temporales de variables meteorológicas (magnitud de la velocidad del viento, humedad relativa y temperatura). Sin embargo, este estudio demuestra que las series de tiempo de concentraciones de $\mathrm{PM}_{10} / \mathrm{PM}_{2.5}$ y CO son caóticas. Lo mismo sucede para las series de tiempo de las variables meteorológicas. Los índices (o cantidades métricas) más importantes en el análisis de series de tiempo no lineales son: tiempo de rezago, $\tau$; dimensión de encajamiento, $\mathrm{m}$; máximo exponente positivo de Lyapunov, $\lambda$; dimensión de correlación, $\mathrm{D}_{\mathrm{C}}$; entropía de Kolmogorov $\mathrm{S}_{\mathrm{K}}$ y el exponente de Hurst, $\mathrm{H}$, que permiten cuantificar el grado de caos del sistema. Entonces, si la dimensión de correlación está bajo 5.0, el exponente de Lyapunov es positivo y la entropía de Kolmogorov tiene un valor finito y positivo. así el caos estaría presente en las series 
CUADRO II. PARÁMETROS PARA ESTUDIO DE CAOS DE TRES VARIABLES DE CONTAMINACIÓN Y TRES METEOROLÓGICAS, EN SEIS ESTACIONES DE MONITOREO (SANTIAGO, CHILE)

\begin{tabular}{|c|c|c|c|c|c|c|}
\hline $\begin{array}{l}\text { Estación } \\
\text { Parámetros }\end{array}$ & $\begin{array}{c}\mathrm{PM}_{10} \\
\left(\mu \mathrm{g} / \mathrm{m}^{3}\right)\end{array}$ & $\begin{array}{c}\mathrm{PM}_{2.5} \\
\left(\mu \mathrm{g} / \mathrm{m}^{3}\right)\end{array}$ & $\begin{array}{l}\mathrm{CO} * \\
(\mathrm{ppm})\end{array}$ & $\begin{array}{c}\text { Temperatura } \\
\left({ }^{\circ} \mathrm{C}\right)\end{array}$ & $\begin{array}{l}\text { HR } \\
(\%)\end{array}$ & $\begin{array}{l}\mathrm{VV} \\
(\mathrm{m} / \mathrm{s})\end{array}$ \\
\hline \multicolumn{7}{|c|}{$E M L$} \\
\hline 1 & 0.491 & 0.603 & 0.514 & 0.440 & 0.613 & 0.777 \\
\hline$D_{c}$ & 4.149 & 4.226 & 3.950 & 2.683 & 3.135 & 4.470 \\
\hline $\mathrm{H}$ & 0.967 & 0.973 & 0.959 & 0.989 & 0.991 & 0.976 \\
\hline $\mathrm{S}_{\mathrm{K}}(1 / \mathrm{h})$ & 0.520 & 0.465 & 0.557 & 0.409 & 0.425 & 0.500 \\
\hline \multicolumn{7}{|c|}{$E M M$} \\
\hline 1 & 0.302 & 0.585 & 0.630 & 0.460 & 0.713 & 0.892 \\
\hline$D_{c}$ & 3.877 & 3.966 & 4.719 & 3.128 & 3.507 & 4.285 \\
\hline $\mathrm{H}$ & 0.972 & 0.977 & 0.981 & 0.991 & 0.990 & 0.980 \\
\hline $\mathrm{S}_{\mathrm{K}}(1 / \mathrm{h})$ & 0.441 & 0.528 & 0.581 & 0.409 & 0.427 & 0.616 \\
\hline \multicolumn{7}{|c|}{$E M N$} \\
\hline 1 & 0.576 & 0.467 & 0.323 & 0.501 & 0.679 & 0.734 \\
\hline$D_{c}$ & 3.952 & 4.019 & 4.332 & 2.951 & 3.496 & 4.239 \\
\hline $\mathrm{H}$ & 0.972 & 0.974 & 0.953 & 0.989 & 0.991 & 0.968 \\
\hline $\mathrm{S}_{\mathrm{K}}(1 / \mathrm{h})$ & 0.463 & 0.545 & 0.523 & 0.426 & 0.366 & 0.470 \\
\hline \multicolumn{7}{|c|}{$E M O$} \\
\hline 1 & 0.467 & 0.289 & 0.229 & 0.453 & 0.689 & 0.855 \\
\hline$D_{c}$ & 3.785 & 4.085 & 4.640 & 2.801 & 3.194 & 4.053 \\
\hline $\mathrm{H}$ & 0.965 & 0.955 & 0.937 & 0.992 & 0.989 & 0.968 \\
\hline $\mathrm{S}_{\mathrm{K}}(1 / \mathrm{h})$ & 0.522 & 0.428 & 0.260 & 0.375 & 0.382 & 0.440 \\
\hline \multicolumn{7}{|c|}{$E M S$} \\
\hline 1 & 0.421 & 0.542 & 0.439 & 0.489 & 0.725 & 0.880 \\
\hline$D_{c}$ & 4.133 & 4.012 & 4.686 & 3.171 & 3.697 & 4.250 \\
\hline $\mathrm{H}$ & 0.969 & 0.973 & 0.953 & 0.990 & 0.992 & 0.957 \\
\hline $\mathrm{S}_{\mathrm{K}}(1 / \mathrm{h})$ & 0.452 & 0.531 & 0.394 & 0.395 & 0.416 & 0.478 \\
\hline \multicolumn{7}{|c|}{$E M V$} \\
\hline 1 & 0.561 & 0.295 & 0.296 & 0.495 & 0.746 & 0.836 \\
\hline $\mathrm{D}_{\mathrm{c}}$ & 3.788 & 3.788 & 4.631 & 3.155 & 3.249 & 3.584 \\
\hline $\mathrm{H}$ & 0.967 & 0.970 & 0.952 & 0.989 & 0.989 & 0.956 \\
\hline $\mathrm{S}_{\mathrm{K}}(1 / \mathrm{h})$ & 0.552 & 0.538 & 0.341 & 0.384 & 0.370 & 0.448 \\
\hline
\end{tabular}

* La dimensión de encajamiento para todas las series fue $\mathrm{m}=3$

CUADRO III. PARÁMETROS NO LINEALES PARA ESTACIÓN DE MONITOREO EMO.

\begin{tabular}{lcccc}
\hline Variable & $\begin{array}{c}\text { Dimensión* } \\
\text { Correlación, }\end{array}$ C & $\begin{array}{c}\text { Exponente } \\
\text { Hurst, H }\end{array}$ & $\begin{array}{c}\text { Exponente } \\
\text { Lyapunov, }\end{array}$ & $\begin{array}{c}\text { Entropía, } \\
\mathrm{S}_{\mathrm{K}} \text { (bits/hora) }\end{array}$ \\
\hline $\mathrm{PM}_{2.5}$ & 3.785 & 0.965 & 0.467 & 0.522 \\
$\mathrm{PM}_{10}$ & 4.085 & 0.955 & 0.289 & 0.428 \\
$\mathrm{CO}$ & 4.640 & 0.937 & 0.229 & 0.260 \\
Temp. & 2.801 & 0.992 & 0.453 & 0.375 \\
HR & 3.194 & 0.989 & 0.689 & 0.382 \\
V.V. & 4.053 & 0.968 & 0.855 & 0.440 \\
\hline
\end{tabular}

* La dimensión de encajamiento para todas las series fue $\mathrm{m}=3$. 
temporales en estudio. La entropía de Kolmogorov $\mathrm{S}_{\mathrm{K}}$, caracteriza el grado de caos para un sistema no lineal. Este valor es proporcional a la tasa de pérdida de la información del sistema.

El exponente de Lyapunov, $\lambda$, caracteriza la naturaleza de la evolución temporal de trayectorias cercanas en el espacio de fase y se considera como componente clave del comportamiento caótico.

Se puede afirmar (Gao et al. 2007) que la entropía de correlación, $\mathrm{K}_{2}$, es una cota inferior de la entropía de Kolmogorov, $\mathrm{S}_{\mathrm{K}}$. Vale decir,

$K_{2} \sim S_{K}$

Estas relaciones forman parte del procedimiento de cálculo numérico a través de un software que se aplica a cada serie temporal (tanto de contaminantes como de variables meteorológicas), cada una de 28464 datos, una vez que no presentan datos faltantes.

\section{RESULTADOS}

El cuadro I presenta la estadística descriptiva de las seis estaciones de monitoreo para concentraciones horarias de partículas $\mathrm{PM}_{2,5}$ en todo el período en estudio.

Además de estas mediciones locales continuas, por sobre tres años, se consideraron variables meteorológicas de temperatura, humedad relativa y velocidad del viento a nivel del suelo. El estudio es localizado debido a las particularidades geográficas y climáticas que inciden sobre la dinámica troposférica del sistema (Salini y Pérez 2015). Sobre estos procesos se superpone otra dinámica más general y compleja de carácter planetario que la hace muy variable debido a su natural conectividad.

En el relleno de series se utilizó el software Golden Surfer (Papritz y Stein 1999, Emery 2005, Ramos et al. 2016). En el estudio no lineal, uno de los parámetros usados fue el exponente de Lyapunov, $\lambda$, usualmente medido en bits por unidad de tiempo (Pesin 1977, Wolf et al. 1985). Otro parámetro es el exponente de Hurst, $\mathrm{H}$, que está relacionado con la suavidad de la curva y con la dimensión fractal, según Mandelbrot (Hurst et al. 1965, Mandelbrot 1975, Geweke y Porter-Hudak 1983, Feder, 1988, Chen et al. 2017). Si el parámetro H está entre 0.5 $\leq H \leq 1.0$, es signo de persistencia (el pasado tiende a persistir en el futuro). Finalmente, la entropía de correlación (entropía de Kolmogorov, ecuación (4), Sprott 2003) $\mathrm{S}_{\mathrm{K}}=\mathrm{dS} / \mathrm{dt}$, para cada variable (es un flujo de entropía medida en bits por unidad de tiempo), se usa como aproximación a la termodinámica según la ecuación (6) y se aplica a las variables de los dos sistemas estudiados (contaminantes y variables meteorológicas de la atmósfera). En el cálculo de algunos parámetros se utilizó el software Chaos Data Analyzer (CDA, Sprott 1995, Sprott 2003). Además, a través del principio de Landauer es posible transformar la entropía de Kolmogorov -medida en bits- a entropía medida en joules/K.

La delgada capa atmosférica en que se centraliza este estudio, tal como se señaló, es del orden de $200 \mathrm{~m}$ snm, de alta densidad, estratificada y recibe el calor transferido por el suelo adyacente que se calienta más rápidamente. En el cuadro II se presentan los valores para las seis estaciones de monitoreo $(\mathrm{Pu}-$ dahuel, Las Condes, Puente Alto, La Florida, Parque O`Higgins y Quilicura), que muestran la evolución temporal de los datos de variables meteorológicas y de concentración de contaminantes.

\section{Estudio de caso: parámetros no lineales en dos estaciones de monitoreo, EMM y EMO}

La figura 6 muestra la evolución temporal del régimen de magnitudes de velocidad del viento en función del tiempo, en las estaciones de monitoreo de Pudahuel (EMO, fondo de valle, serie 1) y Las Condes (EMM, pie de montaña, serie 2). Estas decaen (línea negra) para el periodo de tiempo en estudio, indicando un espacio geográfico menos ventilado. Se puede observar claramente la estacionalidad anual del viento durante el período en estudio.

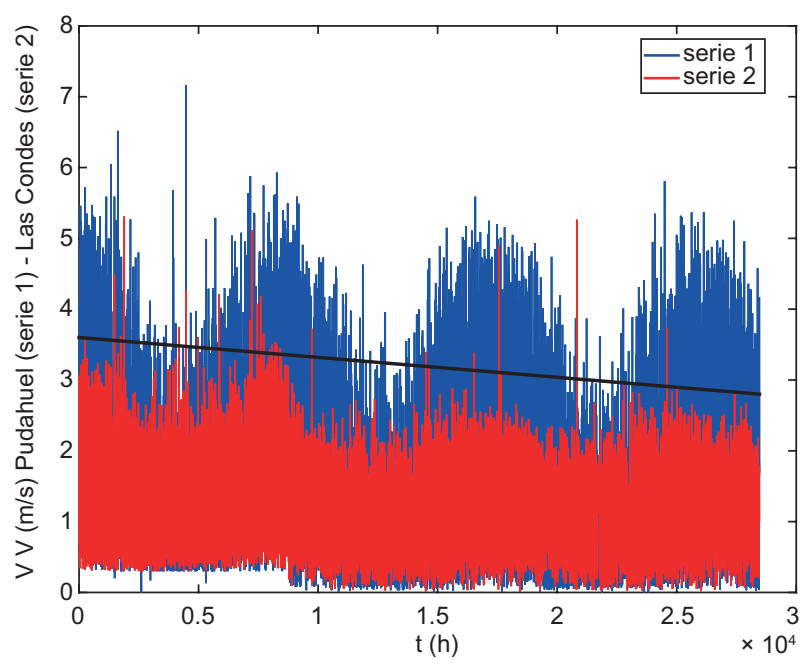

Fig. 6. Evolución temporal de la velocidad del viento para las localidades de Las Condes y Pudahuel. 


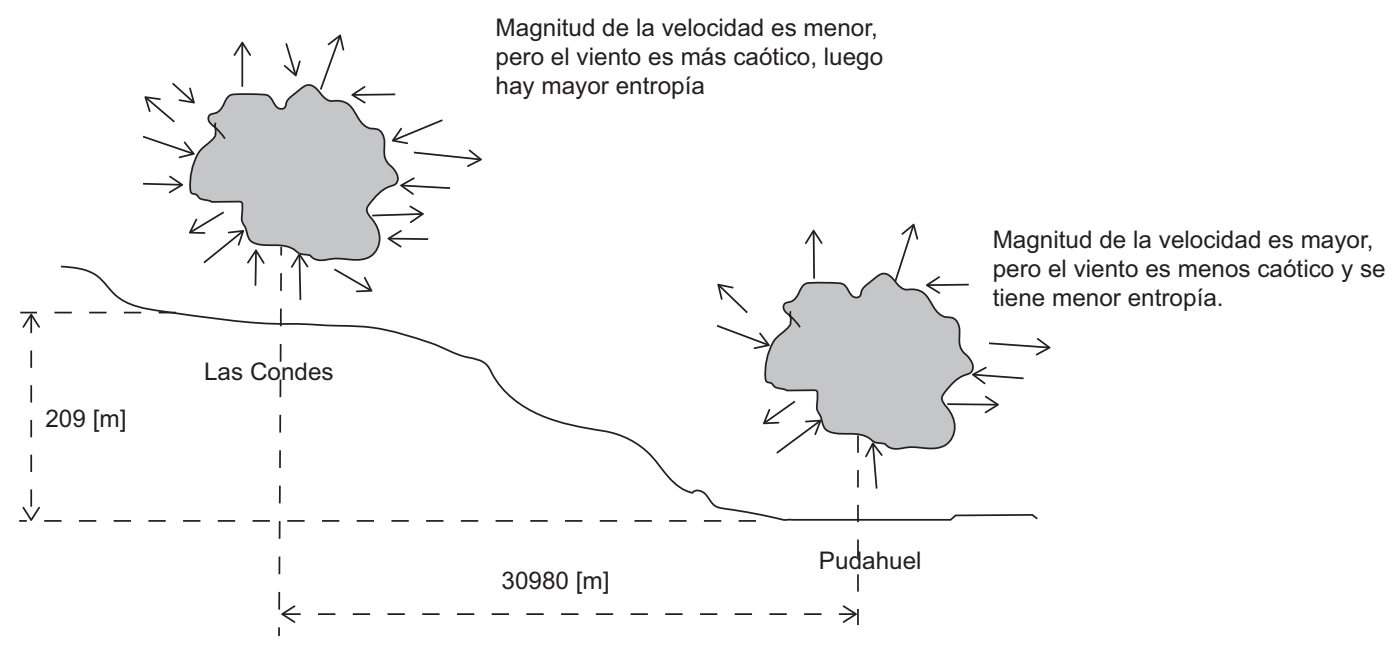

Fig. 7. Diagrama esquemático de dos localidades estudiadas, en Santiago de Chile, considerando regímenes de vientos, elevación y distancia relativa.

La figura 7 presenta un diagrama esquemático de estas dos localidades - distantes entre sí $30.98 \mathrm{~km}-\mathrm{y}$ sus respectivos valores mostrados en cuadros II y IV. Analizando la variable meteorológica de la magnitud de la velocidad del viento (VV), se puede apreciar que la estación EMO tiene el tercer mayor exponente de Lyapunov. Pero presenta la entropía de correlación más baja. En cambio, la estación EMM posee el mayor exponente de Lyapunov y la entropía de correlación más alta. Estos cálculos fueron efectuados empleando software CDA (Sprott 1995).

Es decir, ambos casos presentan caoticidad con baja predictibilidad. Sin embargo, empíricamente la estación EMM exhibe niveles bajos de concentración de los contaminantes estudiados (generados por procesos mecánicos y fotoquímicos) en comparación con estación EMO. Según los resultados de este estudio al comparar, por ejemplo, los regímenes

CUADRO IV. COMPARACIÓN ENTRE SUMA DE VARIABLES DE CONTAMINACIÓN Y METEOROLÓGICAS PARA LA ESTACIÓN EMM.

\begin{tabular}{ccccc}
\hline $\begin{array}{c}\text { Estación } \\
\text { monitoreo }\end{array}$ & $\mathrm{m} \mathrm{snm}^{*}$ & $\begin{array}{c}\text { Distancia }(\mathrm{km}) \\
\text { desde EMN a }\end{array}$ & $\begin{array}{c}\mathrm{S}_{\mathrm{K}}(\mathrm{T}, \mathrm{W}, \\
\mathrm{HR})\end{array}$ & $\begin{array}{c}\mathrm{S}_{\mathrm{K}} \\
\text { (contam.) }\end{array}$ \\
\hline EMO & 469 & 30.98 & 1.197 & 1.210 \\
EMM & 709 & 0 & 1.452 & 1.550 \\
EMS & 485 & 24.78 & 1.289 & 1.377 \\
EML & 784 & 14.24 & 1.334 & 1.542 \\
EMN & 520 & 17.00 & 1.262 & 1.531 \\
EMV & 698 & 20.00 & 1.202 & 1.431 \\
\hline
\end{tabular}

*m snm: metros sobre el nivel del mar. de vientos de EMO con EMM, en esta última localidad los vientos son de menor magnitud, pero más caóticos y por lo mismo de mayor entropía. Lo que se traduce en una mayor movilidad en la traslación de contaminantes

Desde una perspectiva esencialmente entrópica, por los órdenes de magnitud de las entropías de las dos localidades, se favorece el natural flujo de entropía desde la alta entropía de EMM a la baja entropía de EMO, flujo claramente neguentrópico $\left(\mathrm{S}_{\mathrm{K}, \text { FINAL }}-\mathrm{S}_{\mathrm{K}, \text { INICIAL }}<0\right)$. Esto es favorecido por la configuración geográfica y las variables meteorológicas en Santiago de Chile. El régimen de vientos en Las Condes es de menor magnitud en velocidad, pero más caótico, que el de Pudahuel, de mayor magnitud, pero menos caótico, por lo que una fracción del flujo entrópico de contaminación puede orientarse hacia la parte baja del valle donde se ubica Pudahuel.

Como se sabe, la dimensión de correlación, $\mathrm{D}_{\mathrm{C}}$, no debe ser mayor que 5, en caso contrario implicaría datos esencialmente aleatorios (Sprott 2003). Una inspección en la columna correspondiente al cuadro II muestra que ninguna de las bases de datos analizadas está por sobre 5 , entrando todas en la categoría de caótica. Además, en este mismo cuadro se aprecia que todos los exponentes de Lyapunov son positivos y finitos, siendo indicio de caos, lo cual está de acuerdo con un trabajo anterior para la misma ciudad en estudio (Salini y Pérez 2015). En el mismo cuadro II y de acuerdo con los valores del exponente de Hurst y la dimensión de correlación el comportamiento aleatorio se puede descartar. 
La figura 8 representa la suma de los flujos de las entropías de las variables meteorológicas (temperatura $\mathrm{T}$, humedad relativa HR y magnitud de la velocidad del viento VV) versus los flujos de entropías de cada contaminante, $\mathrm{S}_{\mathrm{K} \text {,cont, construidas }}$ a partir de los datos de las mediciones realizadas por las seis estaciones de monitoreo $\left(\mathrm{PM}_{2.5}+\mathrm{CO}+\right.$ $\mathrm{PM}_{10}$ ). Además, en esta misma figura se aprecia la conformación de una banda de flujo entrópico con rango vertical: 1.223 - 1.505 para la entropía de las variables meteorológicas $\left(\mathrm{S}_{\mathrm{K}, \mathrm{VM}}\right)$ y rango horizontal desde 1.290 a 1.651 para el flujo de entropía de los contaminantes ( $\left.\mathrm{S}_{\mathrm{K}, \mathrm{cont}}\right)$.

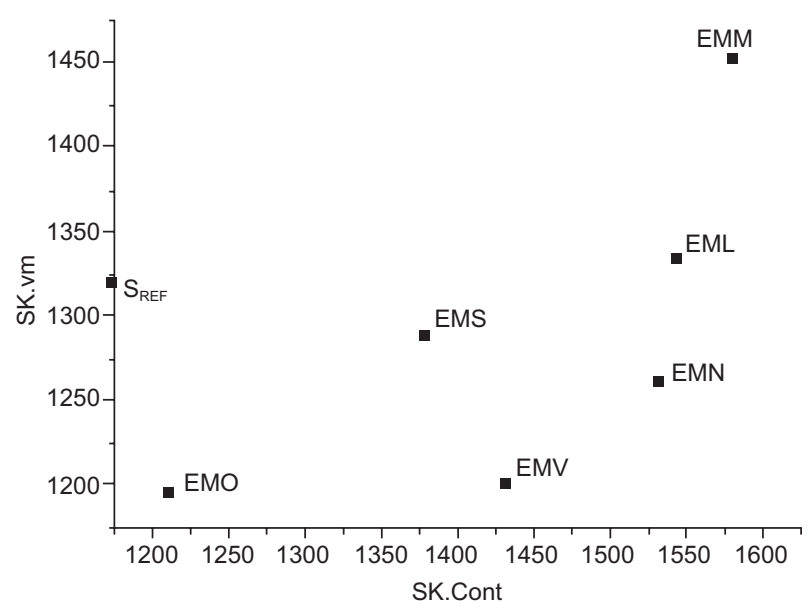

Fig. 8. Entropía de variables meteorológicas, $\mathrm{S}_{\mathrm{vm}}$, versus entropía contaminante, $\mathrm{S}$ cont, según localidad en la que se realizó la medición.

La ecuación $\mathrm{S}_{\mathrm{K} \text {,cont }} \approx 0$, representa el punto de intersección de la serie de tiempo de los pares ordenados $\left(\mathrm{S}_{\mathrm{K}, \text { cont, }} \mathrm{S}_{\mathrm{K}, \mathrm{VM}}\right)$ con el eje vertical de $\mathrm{S}_{\mathrm{K}, \mathrm{VM}}$.

Para la muestra localizada y referida a los datos de las seis estaciones de monitoreo para los contaminantes bajo estudio, este valor se puede interpretar como (i) un sistema con escasa presencia de contaminantes, condición de medio prístino, o (ii) un sistema Tierra-atmósfera troposférico que se sostiene en procesos con características irreversibles $\left(\mathrm{S}_{\mathrm{K}, \mathrm{vm}} \neq\right.$ 0) (Prigogine 1955).

Se puede apreciar que la estación de monitoreo EMM evoluciona espontáneamente desde el equilibrio con máxima entropía hacia otro estado en el que su orden u organización está en una menor entropía, que es el caso de EMO. Así, el proceso entrópico entre estas dos localidades es una manifestación del segundo principio de la termodinámica:
$\Delta S_{K}=S_{K, \text { Pudahuel }}-S_{K, \text { Las Condes }}<0$

el flujo es neguentrópico, lo que permite que el sistema se mantenga fuera del equilibrio, favoreciendo las condiciones desmedradas de ventilación de EMO y propiciando, tal como se indicó, que un porcentaje del flujo entrópico de contaminantes pueda difundirse hacia la parte baja del valle donde se ubica Pudahuel.

\section{Aproximación hacia cero de la entropía de los contaminantes}

De la extrapolación al origen de la serie de tiempo se puede obtener un valor aproximado para flujo de entropía cero (es decir, como un punto de partida) de los contaminantes y con cierta independencia de la localización de la estación de monitoreo (Fig. 8). Esta condición inicial incide en la evolución de la serie de tiempo y su predictibilidad y, además, en la definición de medio prístino. Es decir, en la condición dada están inmersos procesos irreversibles (Prigogine 1967).

Por su parte, la entropía de referencia aproximada para los tres contaminantes $\left(\mathrm{PM}_{2.5}, \mathrm{PM}_{10}\right.$ y CO$)$ se calcula como el promedio de los valores de las variables meteorológicas (eje vertical en Fig. 8) siendo del orden de $\mathrm{S}_{\mathrm{K}, \mathrm{REF}} \approx 1.33$, y se corresponde con $\mathrm{S}_{\mathrm{K} \text {,cont }} \approx 0$ del eje horizontal de contaminantes. $S_{K, R E F}$ señala, desde el punto de vista detallado, ausencia de contaminantes o medio prístino como condición inicial de las variables atmosféricas, cifra que es mayor que la entropía de Kolmogorov particular de cualquier contaminante para todas las localidades estudiadas, lo que es otro indicador del régimen de entropía de Kolmogorov que propicia flujo neguentrópico. En general, se considera que hay un flujo inicial intrusivo desde los contaminantes hacia la atmósfera ya que el promedio de las entropías de Kolmogorov para los valores de los contaminantes es $\mathrm{S}_{\mathrm{K}}$, REF, cont $\approx 1.474$ y se parte de un estado inicial caracterizado por $\mathrm{S}_{\mathrm{K}, \mathrm{REF}} \approx 1.33$. También se confirma un flujo de entropía negativo: $\mathrm{S}_{\mathrm{K}, \operatorname{REF}}(\approx 1.33)-\mathrm{S}_{\mathrm{K}, \mathrm{REF}, \mathrm{cont}}(\approx 1.474)<0$.

La extrapolación hacia el eje vertical del valor medio de los puntos de flujo de entropía de las variables meteorológica en la figura 8, considerada como condición de medio prístino o ausencia de entropía del material contaminante, para las mediciones realizadas se aplica de la siguiente forma:

Para $\mathrm{PM}_{2.5}, \Delta \mathrm{S}_{\mathrm{K}, \mathrm{PM}_{2.5}}=$ flujo de entropía negativa $=$

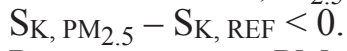

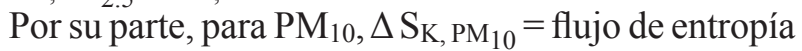
negativa $=\mathrm{S}_{\mathrm{K}, \mathrm{PM}_{10}}-\mathrm{S}_{\mathrm{K}, \mathrm{REF}}<0$.

$\mathrm{Y}$ para $\mathrm{CO} \Delta \mathrm{S}_{\mathrm{K}, \mathrm{CO}}=$ flujo de entropía negativa $=$ $\mathrm{S}_{\mathrm{K}, \mathrm{CO}}-\mathrm{S}_{\mathrm{K}, \mathrm{REF}}<0$. 
Verificándose en los tres casos estudiados un flujo de entropía negativa, que es pérdida de entropía para la atmósfera caracterizada por las variables meteorológicas en condición de entropía de contaminantes cero (medio prístino, Fig. 8).

$\Delta \mathrm{S}_{\mathrm{K}}, \mathrm{PM}_{\text {cont }}=$ flujo de entropía-negativa $=\mathrm{S}_{\mathrm{K}, \text { cont }}-$ $\mathrm{S}_{\mathrm{K}, \mathrm{REF}}<0$.

Esto se puede interpretar como detrimento en las propiedades de la atmósfera (físicas y químicas). Lo que ocurre sin violar la segunda ley de la termodinámica. El cuadro IV, de entropías totales por localidad, corrobora ambas afirmaciones. La primera según diferencia de los datos de las columnas 4 y 5 y la segunda es la suma de los datos de las columnas 4 y 5 (cuyo resultado positivo está en acuerdo con la ecuación (5) para el caso de procesos irreversibles).

El cuadro III, presenta los parámetros no lineales para la estación EMO. En este cuadro y para nuestro estudio de las seis series temporales, se observa que la dimensión de encajamiento, $\mathrm{m}$, se saturó en los valores dados en el cuadro III, indicando presencia de caos, pero no de aleatoriedad. El cuadro IV, exhibe la suma de los flujos de entropías correspondientes a las variables de contaminación y a las variables meteorológicas con distancia referida a EMM. El cuadro $\mathbf{V}$ muestra la suma de los flujos de entropías de las variables meteorológicas $\left(\mathrm{S}_{\mathrm{K}, \mathrm{vm}}\right)$ versus la suma de los flujos de entropía de cada contaminante ( $\mathrm{S}_{\mathrm{K}, \text { cont }}$ ) para EMM y EMO. El cuadro VI, es un resumen comparativo entre las sumas de los flujos de entropías de las variables meteorológicas, $\mathrm{S}_{\mathrm{VM}}$ versus las sumas de los flujos de entropías de los contaminantes,
CUADRO VI. COMPARACIÓN ENTRE SUMA DE ENTROPÍAS DE VARIABLES METEOROLÓGICAS, $\mathrm{S}_{\mathrm{K}, \mathrm{vm}}$, VERSUS SUMA DE ENTROPÍAS DE CONTAMINANTES, $\mathrm{S}_{\mathrm{K}, \mathrm{C}}$, SEGÚN LOCALIZACIÓN DE LA ESTACIÓN DE MONITOREO

\begin{tabular}{lcccc}
\hline $\begin{array}{l}\text { Estación } \\
\text { monitoreo }\end{array}$ & $\mathrm{m} \mathrm{snm}^{*}$ & $\begin{array}{c}\text { Distancia }(\mathrm{km}) \\
\text { desde EMN a }\end{array}$ & $\begin{array}{c}\mathrm{S}_{\mathrm{K}}(\mathrm{T}, \\
\mathrm{W}, \mathrm{HR})\end{array}$ & $\begin{array}{c}\mathrm{S}_{\mathrm{K}, \mathrm{C}} \\
\text { (contam.) }\end{array}$ \\
\hline EMO & 469 & 26.56 & 1.197 & 1.210 \\
EMM & 709 & 17.00 & 1.452 & 1.550 \\
EMS & 485 & 20.00 & 1.289 & 1.377 \\
EML & 784 & 14.00 & 1.334 & 1.542 \\
EMN & 520 & 0 & 1.262 & 1.531 \\
EMV & 698 & 20 & 1.202 & 1.431 \\
\hline
\end{tabular}

*m snm: metros sobre el nivel del mar.

$\mathrm{S}_{\mathrm{K}, \text { cont, }}$ de la estación de monitoreo EMN. Finalmente, el cuadro VII, indica la suma de los valores de los flujos de entropía de variables meteorológicas y de contaminantes para las estaciones, EMM y EMO.

La figura 6 compara las medidas horarias de las magnitudes de las velocidades del viento para EMM y EMO. Estas magnitudes declinan gradualmente en la medida que transcurren los 3.25 años del estudio, condición que influencia la dinámica de los contaminantes. La figura 7 indica las distancias horizontal y vertical entre EMM y EMO junto a los regímenes de viento y su caracterización a partir de sus entropías y coeficientes de Lyapunov. Como en este modelo simplificado, que aplica teoría del caos, la atmósfera se ha caracterizado por las series de tiempo de temperatura, velocidad del viento y humedad relativa, y como a cada una de estas variables se le calcula el

CUADRO V. SUMADE ENTROPÍAS DE VARIABLES METEOROLÓGICAS ( $\left.\mathrm{S}_{\mathrm{K}, \mathrm{vm}}\right)$ VERSUS, SUMA DE ENTROPÍAS PARA CADA CONTAMINANTE $\left(\mathrm{S}_{\mathrm{K}, \mathrm{C}}\right)$

\begin{tabular}{lcccc}
\hline Localidad & $\begin{array}{c}\mathrm{PM}_{2.5} \\
\text { (bits/hora) }\end{array}$ & $\begin{array}{c}\mathrm{PM}_{10} \\
\text { (bits/hora) }\end{array}$ & $\begin{array}{c}\mathrm{CO} \\
\text { (bits/hora) }\end{array}$ & $\begin{array}{c}\text { Entropía variables } \\
\text { meteorológicas (bits/hora) }\end{array}$ \\
\hline EMO & 0.428 & 0.522 & 0.260 & 1.197 \\
EMM & 0.528 & 0.441 & 0.581 & 1.452 \\
\hline
\end{tabular}

CUADRO VII. SUMA DE VALORES DE ENTROPÍA DE VARIABLES METEOROLÓGICAS Y DE CONTAMINANTES PARA ESTACIONES EMM Y EMO

\begin{tabular}{lcccc}
\hline $\begin{array}{l}\text { Estación } \\
\text { monitoreo }\end{array}$ & $\begin{array}{c}\mathrm{PM}_{2.5} \\
\text { (bits/hora) }\end{array}$ & $\begin{array}{c}\mathrm{PM}_{10} \\
\text { (bits/hora) }\end{array}$ & $\begin{array}{c}\mathrm{CO} \\
\text { (bits/hora) }\end{array}$ & $\begin{array}{c}\text { Entropía variables } \\
\text { Meteorológicas (bits/hora) }\end{array}$ \\
\hline EMO & 0.428 & 0.522 & 0.260 & 1.197 \\
EMM & 0.528 & 0.441 & 0.581 & 1.452 \\
\hline
\end{tabular}


flujo de entropía y el flujo entrópico total, entonces, al aplicar igual procedimiento a las series de tiempo de los contaminantes, es posible decidir cuál flujo es el dominante según la localización de las mediciones.

Uno de los propósitos de este trabajo, y que resume la figura 8, es analizar los flujos entrópicos entre la atmósfera y los contaminantes atmosféricos y cual es dominante. Al fin de cuentas el flujo entrópico (bits/unidades de tiempo) es flujo termodinámico vía principio de Landauer mostrando la relación de la atmósfera con el calor. La figura 8 muestra que, para las mediciones locales realizadas, se induce una región de bandas (con máximo y mínimo, tanto para los contaminantes como para las variables meteorológicas), revelando que el flujo entrópico de las variables meteorológicas es más acotado en comparación con los contaminantes. Esto les da a las variables meteorológicas un margen de respuesta limitado a la influencia de los contaminantes en el campo de mediciones locales realizadas.

Para las condiciones localizadas del estudio, se abre la posibilidad, en primera aproximación, de construir un mapa de los flujos de entropías. Esta "huella digital" según condición inicial permitiría estudiar escenarios de eventos.

\section{DISCUSIÓN}

Desde el punto de vista físico para el sistema Tierra-atmósfera troposférico estudiado, la transferencia de su entropía tiene consecuencias en las variables cinéticas con las que se la puede caracterizar. Así, la estación EMO tiene mayor magnitud en la velocidad del viento, pero menor grado de caos y menor entropía. El sistema Tierra-atmósfera dispone de menos entropía a ser sustraída por el contaminante a través del proceso neguentrópico. Esto determina que los elementos contaminantes emitidos, acorde a con su vida media particular, se disipen menos, decantando - una parte de ellos-hacia el suelo con baja dispersión debido a las condiciones atmosféricas desfavorables.

Por el contrario, la estación EMM tiene regímenes de vientos con velocidades de menor magnitud, pero es más caótico y por lo mismo de mayor entropía. Es decir, el contaminante puede "robar" más entropía al sistema Tierra-atmósfera disipándose más y produciéndose, indirectamente, una atmósfera troposférica localizada más limpia.

El análisis según la teoría del caos de 1024704 datos de variables meteorológicas y contaminantes seleccionados en un registro de 3.25 años, y de las entropías y neguentropías calculadas, demuestra que la dinámica que se genera favorece el decaimiento de la concentración del contaminante $\left(\mathrm{PM}_{2.5}, \mathrm{PM}_{10}\right.$, $\mathrm{CO}$ ) en forma gradual o muy lenta permitiendo hablar de su sostenibilidad en el volumen atmosférico estudiado. Esta sostenibilidad no es un a priori, por lo que no se puede definir de entrada, sino que es un resultado al que conducen las condiciones y procedimientos de cálculo particulares del estudio. Debido al factor antrópico que la alimenta, la dinámica asociada al proceso se sostiene en el tiempo en la medida que la tasa de emisión de contaminantes locales tenga cierta regularidad, con claro desmedro de la entropía asociada a las variables meteorológicas propias del sistema Tierra-atmósfera del sector. Es el caso que se presenta en estación EMO y, en general, el de muchas ciudades. Dado el perfil geográfico asociado a las dos localidades en estudio, la estación EMM con su capacidad difusiva contribuye, adicionalmente, al efecto de vertedero de contaminantes en la estación EMO.

Finalmente, las series de tiempo de los contaminantes en estudio presentan signos de caos, confirmando una de nuestras hipótesis. Esto está en concordancia con el cuadro II, donde todos los exponentes de Lyapunov son positivos.

\section{CONCLUSIONES}

Este estudio muestra que es posible analizar la difusión de un sistema de contaminantes a través del sistema Tierra-atmósfera, aplicándolo al estrato atmosférico inmediatamente adyacente al suelo, a partir de las entropías características del sistema Tierra-atmósfera altamente entrópico. Se conforma una dinámica de sumidero, la que se explica por la neguentropía y su flujo asociado con el incremento de la concentración y densidad de contaminantes debido a las emisiones locales y condiciones meteorológicas adversas para una localidad geográfica o de fuente (a modo de un emisor que contribuye a la propagación del contaminante dispersándolo más allá de su zona de origen haciendo más intenso el efecto sumidero para dos localidades relativamente adyacentes). La observación en el terreno de estas características es una confirmación, en primera aproximación, de lo expuesto. Si bien es cierto que la dispersión de los contaminantes se desplazaría verticalmente bajo condiciones meteorológicas inestables o turbulentas, cuando existen episodios de contaminación la estabilidad atmosférica (como la inversión térmica) dificulta su dispersión vertical, lo que contribuye a su acumulación cerca de las fuentes emisoras. En 
este estudio hemos hallado que los contaminantes emitidos en estación EMM pueden desplazarse hasta la estación EMO.

El resultado encontrado más relevante es que, físicamente, un contaminante se puede volver sostenible gracias al aporte antrópico. Vale decir, el cambio negativo de entropía permite que un sistema se pueda mantener en el tiempo, lejos del equilibrio, contribuyendo a su dispersión durante el desplazamiento.

Los valores obtenidos para los exponentes de Lyapunov, exponente de Hurst y dimensión de correlación, permiten descartar un comportamiento aleatorio, concluyéndose que las series de tiempo son caóticas y muy sensibles a las condicione iniciales.

Finalmente, hemos corroborado que los contaminante $\mathrm{PM}_{2.5}$ y $\mathrm{PM}_{10}$ se comportan de manera caótica, tal como fue sugerido y demostrado por Salini y Pérez (2015). Se requerirá un estudio cuantitativo adicional para aseverar de una manera más convincente esta última conclusión, como, por ejemplo, el análisis de cuantificación recurrente (RQA, por sus siglas en inglés) el cual es un método de análisis de datos no lineales que cuantifica el número y duración de recurrencias de un sistema dinámico representado por su trayectoria en el espacio de las fases.

\section{AGRADECIMIENTOS}

Este trabajo fue parcialmente financiado por los proyectos DIN 13/2016 y DIREG 04/2019 de la Dirección de Investigación e Innovación, y la Facultad de Ingeniería de la Universidad Católica de la Santísima Concepción. Los autores también desean agradecer a la Dirección de Investigación que financió este estudio a traves del Proyecto LPR2002 y al Departamento de Física pertenecientes a la Universidad Tecnológica Metropolitana.

\section{REFERENCIAS}

Araujo J.A. (2011). Particulate air pollution, systemic oxidative stress, inflammation, and atherosclerosis. Air Qual. Atmos. Health 4, 79-93. https://doi.org/10.1007/ s11869-010-0101-8

Asa E., Saafi M., Membah J. y Billa A. (2012). Comparison of linear and nonlinear Kriging methods for characterization and interpolation of soil data. J. Comput. Civil Eng. 26(1), 11-18. https://doi.org/10.1061/(ASCE) CP.1943-5487.0000118.

Belcik M.K., Trusz-Zdybek A., Zaczynska E., Czarny A. and Piekarska K. (2018). Genotoxic and cyto- toxic properties of $\mathrm{PM}_{2.5}$ collected over the year in Wroclaw (Poland). Sci. Total Environ. Oct (1), 637-638: 480-497. https://doi.org/10.1016/j.scitotenv.2018.04.166

Bénard H. (1900). Tourbillons cellulaires dans une nappe liquid. Revue Générale des Sciences Pures et Appliquées 11, 1261-1271, 1309-1328.

Bénard H. (1901). Les tourbillons cellulaires dans une nappe liquide propageant de la chaleur par convection en régime permanent. Ann. Chim. Phys. 23, 62-164. https://doi.org/10.1007/BF01703351

Biblioteca del Congreso Nacional de Chile [en línea]. https: //www.bcn.cl/ 01/06/2019

Brillouin L. (1962). Science and Information Theory. 2a ed. Academic Press, Nueva York, 304 pp .

Chen Y., Wang J. y Feng J. (2017). Uunderstanding the fractal dimensions of urban forms through spatial entropy. Entropy 19 (11), 600. https://doi.org/10.3390/ e19110600

Chelani A. y Devotta S. (2006). Nonlinear analysis and prediction of coarse particualte matter concentration in ambient air. J. Air Waste Manag. Asoc. 56, 78-84. https://doi.org/10.1080/10473289.2006.10464432

Cifuentes L. A., Vega J., Köpfer K. y Lave L. B. (2000). Effect of the fine fraction of particulate matter versus the coarse mass and other pollutants on daily mortality in Santiago, Chile. J. Air Waste Manag. Assoc. 50(8), 1287-1298.https://doi.org/10.1080/10473289.2000. 10464167

Cohen A. y Procaccia I. (1985). Computing the Kolmogorov entropy from time signals of dissipative and conservative dynamical systems. Phys. Rev. A Gen. Phys. 31 (3), 1872-1882. https://doi.org/10.1103/ PhysRevA.31.1872

Cressie N. y Huang H. (1999). Classes of no separable spatio temporal stationary covarianza function. J. Am. Stat. Assoc. 94:1330-1340.https://doi.org/10.2307/2669946

Cuesta J.A. (2006). La entropía como creadora de orden. Rev. Esp. Física. 20, 13-19.

De Groot S.R. y Mazur P. (1984). Non-equilibrtium thermodynamics. Dover Publications, Mineola, Nueva York, EUA. 510 pp.

Dewar R.C. (2005). Maximum entropy production and the fluctuation theorem. J. Phys. A: Math. Gen. 38, 1371-1381. https://doi.org/10.1088/0305-4470/38/21/ L01.

Eckert K., Bestehorn M. y Thess A. (1998). Square cells in surface-tension-driven Bénard convection: experiment and theory. J. Fluid Mech., 356, 155-197. https://doi. org/10.1017/S0022112097007842

Eckmann J. P., Oliffson S., Kamphorst S., Ruelle D. y Ciliberto C. (1986). Lyapunov exponents from time series. Phys. Rev. A 34, 4971-4979. https://doi.org/10.1007/ 


\section{$\mathrm{BFb} 0086675$}

Emery X. (2005). Simple and ordinary multigaussian Kriging for estimating recoverable reserves. Math. Geol. 37, 295-319. https://doi.org/10.1007/s11004005-1560-6.

Farmer J.D. (1982). Chaotic attractors of an infinitedimensional dynamical system. Physica 4D, 366-393. https://doi.org/10.1016/0167-2789(82)90042-2

Farmer J.D., Otto E. y Yorke J.A. (1983). The dimension of chaotic attractors. Physica D7, 153-180. https://doi. org/10.1016/0167-2789(83)90125-2

Feder J. (1988). Fractals. Springer, Nueva York, EUA, $284 \mathrm{pp}$.

Fraser A. M. y Swinney H. L. (1986). Independent coordinates for strange attractors from mutual information. Phys. Rev. A 33, 1134-1140. DOI:10.1103/ PhysRevA.33.1134

Gao J., Cao Y. y Hu J. (2007). Multiscale analysis of complex time series. Wiley and Sons Interscience, Nueva Jersey, EUA, 368 pp.

Geweke J. y Porter-Hudak S. (1983). The estimation and application of long memory time series models. J. Time Ser. Anal. 4, 221-238. https://doi. org/10.1111/j.1467-9892. 1983.tb00371.x

Glansdorff P. y Prigogine I. (1971). Thermodynamic theory of structure, stability and fluctuations. Wiley and Sons Interscience, Nueva York, EUA, 232 pp.

Grassberger P. y Procaccia L. (1983). Characterization of strange attractors. Phys. Rev. Lett. 50, 346-349. https:// doi.org/10.1103/PhysRevLett.50.346

Haddad W. (2017). Thermodynamics: The Unique Universal Science. Entropy 19 (11), 621. DOI:10.3390/ e19110621

Hurst H.E., Black R.P. y Simaika Y.M. (1965). Long-term storage: an experimental study. Constable, Londres, Reino Unido, 165 pp.

Ilabaca M., Olaeta I., Campos E., Villaire J., Telles-Rojo M. y Romieu, I. (1999). Association between levels of fine particulate and emergency visits for pneumonia and other respiratory illnesses among children in Santiago, Chile. J. Air Waste Manag. Assoc. 49, 154-163. https://doi.org/10.1080/10473289.1999.10463879

Jia Y., Wang Q. y Liu T. (2017). Toxicity research of $\mathrm{PM}_{2.5}$ compositions in vitro. Int. J. Environ. Res. Public Health, 14(3), 232-249. https://doi.org/10.3390/ ijerph14030232.

Junninen H., Niska H., Tuppurainen K., Ruuskanen J. y Kolehmainen M. (2004). Methods for imputation of missing values in air quality data sets. Atmos. Environ. 38, 2895-2907. https://doi.org/10.1016/j. atmosenv.2004.02.026

Kantz H. y Schreiber T. (2004). Nonlinear time series analysis. $2 \mathrm{a}$ ed. Cambridge University Press, Cam- bridge, Reino Unido, 387 pp.

Katchalsky A. y Curran P.F. (1965). Nonequilibrium thermodynamics in biophysics. Harvard University Press, Cambridge, MA., EUA, 248 pp.

Kyriakidis P. y Journel A. (1999). Geostatistical space-time models: A review. Math. Geol. 6, 651-684. https://doi. org/10.1023/A:1007528426688

Kolmogorov A. N. (1959). On entropy per unit time as a metric invariant of automorphisms. Dokl. Akad. Nauk SSSR 124, 754-755.

Kumar U., Prakash A. y Jain V.K. (2008). Characterization of chaos in air pollutants: a Volterra-Wiener-Korenberg series and numerical titration approach. Atmos. Environ. 42, 1537-1551. https://doi.org/10.1016/j. atmosenv.2007.10.076

Lee C. K. y Lin S. C. (2008). Chaos in air pollutant concentration (APC) time series. Aerosol Air Qual. Res. 8, 381-391. https://doi.org/10.4209/aaqr.2008.09.0039

Lee B., Kim B. y Lee K. (2014). Air pollution exposure and cardiovascular disease. Toxicol. Res. 30 (2), 71-75. https://doi.org/10.5487/TR.2014.30.2.071.

Liu Y., Liu Ch. y Wang D. (2011). Understanding atmospheric behaviour in terms of entropy: A review of applications of the second law of thermodynamics to meteorology. Entropy 13, 211-240. https://doi. org/10.3390/e13010211.

López-Corona O., Padilla P., Huerta A., Mustri-Trejo D., Pérez K. Ruiz A., Valdés O. y Zamudio F. (2016). Measuring social complexity and the emergence of cooperation from entropic principles. $6 \mathrm{pp}$. arXiv:1502.05741v2 [physics.soc-ph].

Lorenz E. (1963). Deterministic nonperiodic flow. J Atmos. Sci. 20, 130-141. https://doi.org/10.1175/15200469(1963)020<0130:DNF>2.0.CO;2

Mandelbrot B.B. (1975). Stochastic models for the Earth's relief, the shape and fractal dimension of the coastline, and the number-area rule for islands. P. Natl. Acad. Sci. USA 72 (10), 3825-3828. https://doi.org/10.1073/ pnas. 72.10 .3825

Manríquez R. (1987). Estructuras disipativas. De la termodinámica a la psicoterapia familiar. Rev. Asoc. Esp. Neuropsiquiatría VII (11), 435-454.

Martínez J.A. y Vinagre F.A. (2016). La entropía de Kolmogorov; su sentido físico y su aplicación al estudio de lechos fluidizados 2D. Departamento de Química Analítica e Ingeniería Química, Universidad de Alcalá, Alcalá de Henares, Madrid, España [en línea]. https:// www.academia.edu/2479372 19/07/2019

Mahulikar S.P. y Herwig H. (2009). Exact thermodynamic principles for dynamic order existence and evolution in chaos. Chaos Soliton. Fract. 4 (4), 1939-1948. https:// doi.org/10.1016/j.chaos.2008.07.051.

Mera E., Cordero, B., Condal A., Gutiérrez L., Ríos C, Da 
Silva L. (2018). Filling of missing data in atmospheric series with linear krigeage. J. Phys. Conf. Ser. 1043 (1), 1-6. https://doi.org/10.1088/1742-6596/1043/1/012064.

MMA (2019) Sistema de Información Nacional de Calidad del Aire. [en línea] https://sinca.mma.gob.cl/index.php (Ministerio del Medioambiente de Chile). 30/04/2019.

Molina M. J. y Molina L. T. (2004). Megacities and atmospheric pollution. J. Air Waste Manag. Assoc. 54, 10473289. https://doi.org/10.1080/10473289.2004.10470936.

Morales R. G. E. (2006). Contaminación atmosférica urbana: episodios críticos de contaminación ambiental en la ciudad de Santiago. Editorial Universitaria, Santiago, Chile, 327 pp.

Norazian M. N., Shruki Y. A., Azam R. M. y Mustafa Al Bakri A. M. (2008). Estimation of missing values in air pollution data using single imputation techniques. ScienceAsia 34, 341-345. https://doi.org/10.2306/ scienceasia1513-1874.2008.34.341

OMS (2018) Guías de calidad del aire de la OMS relativas al material particulado, el ozono, el dióxido de nitrógeno y el dióxido de azufre. Organización Mundial de la Salud, Ginebra, Suiza. [en línea] https://www. who.int/es/news-room/fact-sheets/detail/ambient(outdoor)-air-quality-and-health 27/03/2019

Onsager L. (1931) Reciprocal relations in irreversible processes.I. Phys. Rev. 37, 405-426. https://doi. org/10.1103/PhysRev.37.405

Papritz A. y Stein A. (1999). Spatial prediction by linear kriging. En: Spatial statistics for remote sensing (Stein A., van der Meer F. D. y Gorte B.G.H., Eds.). Kluwer Academic, pp. 83-113, Doordrecht, Países Bajos.

Pesin Y.B. (1977). Characteristic Lyapunov exponents and smooth ergodic theory. Russ Math Surv+ 32 (4), 55-114. https://doi.org/10.1070/RM1977v032n04ABEH001639

Peters A. y Dockery D.W. (2005). Lung biology in health and disease. En: Air pollutants and the respiratory tract. (W.M. Foster y D.L. Costa, Eds.) CRC Press, pp.1-20. Boca Raton, EUA,

Prigogine I. (1955). Introduction to Thermodynamics of Irreversible Processes. Charles C. Thomas, Springfield, EUA, $115 \mathrm{pp}$

Prigogine I. (1967). Introduction to thermodynamics of irreversible processes. Interscience. Nueva York, EUA, 147 pp.

Raabe O. G. (2005). Respiratory exposure to air pollutants. En: Air pollutants and the respiratory tract (D.L. Swift y W.M. Foster, Eds.) Taylor and Francis, Boca Ratón, E.U.A. pp. 39-74.

Ramos Y., St-Onge B., Blanchet J.P. y Smargiassi A. (2016). Spatio-temporal models to estimate daily concentrations of fine particulate matter in Montreal: Kriging with external drift and inverse distance-weighted approaches. J. Expo. Sci. Env. Epid. 26 (4), 405-414. https://doi.org/10.1038/jes.2015.79

Reyna M.A., Mérida J.V., Osornio-Vargas A.R., Lerma C., Bravo-Zanoguera M.E., Avitia, R.L. y Nieblas E.C. (2018). Association between personal $\mathrm{PM}_{10}$ exposure and pulmonary function in healthy volunteers from a semi-arid city on the US-Mexican border. Rev. Int. Contam. Ambie. 34 (4), 583-585. https://doi. org/10.20937/RICA.2018.34.04.03

Ruelle D.P. (2003). Extending the definition of entropy to non-equilibrium steady states. P. Natl. Acad. Sci. USA 100 (6), 3054-3058. https://doi.org/10.1073/ pnas.0630567100.

Rutland J. y Garreaud R. (1995). Meteorological air pollution for Santiago, Chile: towards an objective episode forecasting. Environ. Monit. Asess. 34:223-244. https://doi.org/10.1007/BF00554796

Salini G. y Pérez P. (2006). Estudio de series temporales de contaminación ambiental mediante técnicas de redes neuronales artificiales. Ingeniare 14, 284-290. https:// doi.org/10.4067/S0718-33052006000200012

Salini G. (2014). Estudio acerca del material particulado emitido en ciudades de tamaño medio al sur de Santiago de Chile. INGE CUC 10 (1), 97-108.

Salini G. y Pérez P. (2015). A study of the dynamic behavior of fine particulate matter in Santiago, Chile. Aerosol Air Qual. Res. 15, 154-165.https://doi.org/10.4209/ aaqr.2013.10.0323

Salini G.A. y Medina E. (2017). Estudio sobre la dinámica temporal de material particulado $\mathrm{PM}_{10}$ emitido en Cochabamba, Bolivia. Rev. Int. Contam. Ambie. 33 (3), 437-448. https://doi.org/10.20937/RICA.2017.33.03.07

Schrödinger E. (1944). What is life? The physical aspect of the living cell. Cambridge University Press, Londres, Reino Unido, $32 \mathrm{pp}$.

Schwartz J., Laden F. y Zanobetti A. (2002). The concentration-response relation between $\mathrm{PM}_{2.5}$ and daily deaths. Environ. Health Perspec. 110, 1025-1029. https://doi.org/10.1289/ehp.021101025

Shannon C. (1948). A mathematical theory of communication. Bell System Technical Journal 27(3), 379-423 y 623-656. https://doi.org/10.1002/j.1538-7305.1948. tb01338.x

Shaw R. (1981). Strange attractors, chaotic behavior and information flow. Z Naturforsch A 36, 80-112. https:// doi.org/10.1515/zna-1981-0115

Sinai Y. G. (1959). On the concept of entropy of a dynamical system. Dokl. Akad. Nauk SSSR 124, 768-771.

Sivakumar B., Wallender W., Horwath W. y Mitchell J. (2007). Nonlinear deterministic analysis of air pollution dynamics in a rural and agricultural setting. Adv. Complex Syst. 10, 581-597. https://doi.org/10.1142/ S0219525907001288

Sprott J. C. (1995). Chaos Data Analyzer software. http:// 
sprott.physics.wisc.edu/cda.htm

Sprott J. C. (2003). Chaos and time-series analysis. Oxford University Press, Oxford, Reino Unido, 528 pp.

Stephens G.L. y O`Brien D.M. (1993). Entropy and climate. I: ERBE observations of the entropy production. Quart. J. Roy. Meteorol. Soc. 119, 121-152. https://doi. org/10.1002/qj.49711950906

Takens F. (1981). Detecting strange attractors in turbulence. En: Dynamical Systems and Turbulence, Warwick 1980 ( D. Rand y LS.Young, Eds). Springer, Berlin, Alemania, pp. 366-381. https://doi.org/https://
doi.org/10.1007/BFb0091924

Toledano C. (2008). Los aerosoles atmosféricos y su influencia en la península ibérica. Manual formativo de ACTA No 48. [en línea] https://dialnet.unirioja.es/ servlet/articulo? codigo $=5098746$ 21/06/2019

Torday J.S. (2016). Life is simple - biologic complexity is an epiphenomenon. Biology 5 (2), 17. https://doi. org/10.3390/biology5020017

Wolf A., Swift J.B., Swinney H.L. y Vastano J.A. (1985). Determining Lyapunov exponents from a time series. Physica D 16, 285-317. https://doi.org/10.1016/01672789(85)90011-9 


\section{ANEXO I}

\section{Equivalencia entre distintas entropías y paráme- tros asociados}

Usando la Primera Ley de la Termodinámica, la ecuación de continuidad y la ecuación de balance por componentes y reescribiendo la derivada local de $\mathrm{S}=$ $\mathrm{V} \times \mathrm{s}$, donde $\mathrm{V}$ es el volumen, se determina la razón de emisión de entropía por unidad de volumen, s, a partir de la ecuación (1):

$$
\begin{aligned}
& \frac{\partial S}{\partial t}=-\nabla \cdot\left(S \vec{v}+\frac{1}{T} \vec{J}_{\text {Flujo de calor }}-\right. \\
& \left.\sum_{j=1}^{r} \frac{\mu_{j}}{T} \vec{J}_{\text {Flujo difusivo de masa }, j}\right)+\sigma
\end{aligned}
$$

Según Onsager (Onsager 1931), la componente j del flujo de calor es:

$$
\begin{gathered}
J_{\text {Flujo de calor }, j}=J_{q, j}=-K \frac{\partial T}{\partial x_{j}} \\
\text { unidades S.I } \therefore\left[\frac{\text { Joules }}{\operatorname{seg} m k}\right]\left[\frac{K}{m}\right]
\end{gathered}
$$

En esta ecuación, K es la conductividad térmica, mientras que

$$
\begin{array}{r}
\vec{J}_{\text {Fhjo difusivo de masa } a_{j}}=\vec{J}_{m, j}=\rho_{r j}\left(\overrightarrow{v_{j}}-\vec{v}\right) \\
\text { unidades S } . I .:\left[\frac{\mathrm{mol}}{\mathrm{m}^{3}}\right]\left[\frac{\mathrm{m}}{\mathrm{seg}}\right]
\end{array}
$$

donde $\vec{v}_{j}$ es la componente $\mathrm{j}$ de la velocidad relacionada a $v=\sum_{j=1}^{r} \rho_{j} \vec{v}_{j} / \rho, \rho_{r j}$ es cantidad de moles de la especie $\mathrm{j}$ en el volumen $\mathrm{V}$ y se expresa en moles en metros cúbicos y a $\sigma$ se le denomina término de producción de entropía a los alrededores con unidades de Potencia/(Temperatura•Volumen).

Análisis de unidades en el sistema internacional para ecuación (11). A partir de dicha ecuación

se desprende que las unidades de medida son:

$$
\begin{aligned}
& \frac{1}{\operatorname{seg}} \frac{J}{K}\left[\frac{1}{m^{3}}\right] \equiv \frac{1}{m} \frac{J}{K}\left[\frac{m}{\operatorname{seg}}\right] \frac{1}{m^{3}}+\left[\frac{1}{m}\right] \frac{1}{K} \frac{J}{K m s e g} \\
& {\left[\frac{K}{m}\right]+\left[\frac{1}{m}\right]\left[\frac{1}{K}\right]\left[\frac{J}{m o l}\right]\left[\frac{m o l}{m^{3}}\right]\left[\frac{m}{s e g}\right]+\frac{1}{s e g} \frac{J}{K}\left[\frac{1}{m^{3}}\right]}
\end{aligned}
$$

\section{La expresión:}

$-\nabla \cdot(S \vec{v})$

representa el flujo de entropía asociado a la configuración de las partículas constituyentes del sistema y se relaciona con sus posiciones ya sea por sus velocidades o momentum. Está físicamente ligada con el número de arreglos posibles en que pueden tenerse todas las partículas mientras se mantienen conjuntos de propiedades del sistema especificado, como la energía.

Al escribir:

$T \frac{\partial s}{\partial t}=-\nabla \cdot\left(\vec{J}_{\text {Flujo de calor }}\right)$

se tiene la tasa de acumulación local de calor.

El término:

$-\nabla \cdot\left(\frac{1}{T} \vec{J}_{\text {Flujo de calor }}\right)$

es la razón de incremento de entropía local por unidad de volumen y el término:

$-\nabla \cdot\left(\sum_{j=1}^{r} \frac{\mu_{j}}{T} \vec{J}_{\text {Flujo difusivo de masa }, j}\right)$

representa la variación -en el tiempo-de la entropía por unidad de volumen asociada al potencial químico para todas las especies componentes.

Finalmente, se construye el flujo de entropía, $\vec{J}_{s}$ a partir de la ecuación (2):

$\overrightarrow{J_{S}}=s \vec{v}+\frac{1}{T} \vec{J}_{q}-\sum_{j=1}^{r} \frac{\mu_{j}}{T} \vec{J}_{j}$

(en unidades del Sistema Internacional: $\mathrm{J} /\left(\operatorname{seg} \mathrm{K} \mathrm{m}^{2}\right.$ ) $\left.=\left(\mathrm{W} / \mathrm{m}^{2}\right) / \mathrm{K}\right)$.

Concluyendo, así, la ecuación (1):

$\frac{\partial S}{\partial t}=-\nabla \cdot \overrightarrow{J_{S}}+\sigma$

donde:

$-\nabla \cdot \overrightarrow{J_{S}}$

es el flujo de entropía por unidad de volumen, a través de la superficie cuyo contorno encierra al sistema. 